\title{
O uso da telessaúde em tempos
}

\section{de pandemia}

\author{
Kleize Araújo de Oliveira Souza, ${ }^{1}$ Elzo Pereira Pinto Junior, ${ }^{2}$ Rafael \\ Damasceno de Barros, ${ }^{3}$ Acácia Mayra Pereira de Lima, ${ }^{4}$ Nathália \\ de Oliveira Silva, ${ }^{5}$ Lizeth Yubalena Orozco Beltrán, ${ }^{6}$ Luis Eugenio \\ Portela Fernandes de Souza
}

1 Enfermeira. Doutora em Saúde Pública pelo Instituto de Saúde Coletiva (ISC) da Universidade Federal da Bahia (UFBA). Professora adjunta da Universidade Estadual de Feira de Santana (UEFS). Pesquisadora do Núcleo de Pesquisa Integrada em Saúde Coletiva (Nupisc) da UEFS. Pesquisadora colaboradora da Rede CoVida da Fundação Oswaldo Cruz (Fiocruz) e da UFBA.

2 Fisioterapeuta. Doutor em Saúde Pública pelo Instituto de Saúde Coletiva (ISC) da Universidade Federal da Bahia (UFBA). Pesquisador do Centro de Integração de Dados e Conhecimentos para a Saúde (Cidacs) da Fundação Oswaldo Cruz (Fiocruz). Pesquisador colaborador da Rede CoVida da Fiocruz e da UFBA.

3 Enfermeiro. Mestre em Saúde Comunitária pelo Instituto de Saúde Coletiva (ISC) da Universidade Federal da Bahia (UFBA). Professor assistente da UFBA. Pesquisador do Programa Integrado de Pesquisa e Cooperação Técnica em Economia, Tecnologia e Inovação em Saúde (PECS) da UFBA. Pesquisador colaborador da Rede CoVida da Fundação Oswaldo Cruz (Fiocruz) e da UFBA.

4 Assistente Social. Mestra em Saúde Comunitária pelo Instituto de Saúde Coletiva (ISC) da Universidade Federal da Bahia (UFBA). Pesquisadora do Núcleo de Ensino e Pesquisa em Aids e outras Doenças Infecciosas (Nepadi) da UFBA. Pesquisadora colaboradora da Rede CoVida da Fundação Oswaldo Cruz (Fiocruz) e da UFBA.

5 Graduanda em Psicologia pela Universidade Estadual de Feira de Santana (UEFS). Integrante do Núcleo de Pesquisa Integrada em Saúde Coletiva (Nupisc) da UEFS.

6 Médica. Doutoranda em Saúde Pública pelo Instituto de Saúde Coletiva (ISC) da Universidade Federal da Bahia (UFBA).

7 Sanitarista. Professor do Instituto de Saúde Coletiva (ISC) da Universidade Federal da Bahia (UFBA). Membro da coordenação da Rede CoVida da Fundação Oswaldo Cruz (Fiocruz) e da UFBA.

SOUZA, K. A. de O.; PINTO JUNIOR, E. P.; BARROS, R. D. de; LIMA, A. M. P. de; SILVA, N. de O.; BELTRÁN, L. Y. O.; SOUZA, L. E. P. F. de. O uso da telessaúde em tempos de pandemia. In: BARRETO, M. L.; PINTO JUNIOR, E. P.; ARAGÃO, E.; BARRAL-NETTO, M. (org.). Construção de conhecimento no curso da pandemia de COVID-19: aspectos biomédicos, clínico-assistenciais, epidemiológicos e sociais. Salvador: Edufba, 2020. v. 2. DOI: https://doi.org/10.9771/9786556300757.019 


\section{Introdução}

A pandemia de COVID-19 tem exigido adaptações em muitos comportamentos e práticas, alguns socialmente cristalizados, para evitar a disseminação da doença. As medidas de distanciamento social, que representam uma das principais estratégias para o combate ao novo coronavírus, desafiam o cotidiano de bilhões de pessoas pelo mundo e afetam diretamente a organização dos serviços de saúde.

Nesse cenário, a Telessaúde (TS) e a Telemedicina (TM) surgem como ferramentas potencialmente capazes de garantir os cuidados em saúde em um cenário em que se faz imperativo evitar a desnecessária circulação de pessoas. Este capítulo tem como objetivo apresentar, baseando-se em revisão da literatura nacional e internacional, os conceitos, as aplicações e os desafios do uso da telessaúde no combate à pandemia de COVID-19 e fazer recomendações relativas à adoção da TS pelos serviços de saúde no Brasil.

Em 11 de março de 2020, a Organização Mundial da Saúde (OMS) declarou que a doença causada pelo SARS-CoV-2 tinha alcançado o estágio de pandemia. Desde então, o número de regiões e países afetados 
cresceu muito, impondo grandes desafios a todas as sociedades, incluindo os sistemas de saúde.

A COVID-19 é uma doença para a qual, até setembro de 2020, não existem remédios ou vacinas com comprovada eficácia. Seu enfrentamento, portanto, exige a adoção de um conjunto de medidas não farmacológicas. Dentre as estratégias globais de combate à pandemia, destacam-se a prevenção de casos através de higiene das mãos, etiqueta respiratória, distanciamento físico em nível individual, isolamento dos casos e quarentena dos contatos, além de restrições de viagens domésticas e internacionais. (WHO, 2020)

Quando o movimento de pessoas é restringido em todo o mundo e cidades ou regiões inteiras são sujeitas a medidas de distanciamento social, seja ele obrigatório ou não, os indivíduos afetados estão suscetíveis a aumento do estresse da vida diária, encargos econômicos inesperados, exposição a outras doenças transmissíveis e não transmissíveis e vários transtornos de ordem psicológica. (CHAUHAN et al. 2020; PALADINO et al., 2017 PAPADIMOS et al., 2018;) Consequentemente, soluções inovadoras são necessárias para ajudar a atender às necessidades não apenas dos pacientes de COVID-19, mas também de todos os outros que possam apresentar alguma necessidade de atenção à saúde. (GREENHALGH, 2020)

Sob tais condições, a TS pode representar uma importante estratégia tanto para o acompanhamento de pacientes em fase de quarentena, quanto pra a triagem de novos casos e identificação daqueles que necessitam de uma avaliação presencial.

\section{Alguns conceitos importantes}

O desenvolvimento de novas tecnologias digitais impõe discussões relevantes para o cuidado da saúde da população no contexto 
atual de uma pandemia, pois traz a possibilidade de promover mudanças que permitam aos serviços de saúde prover cuidados, aproveitando as vantagens do uso da internet e das telecomunicações em geral. Considerando a rapidez das mudanças tecnológicas e de suas aplicações à assistência em saúde, um primeiro desafio se refere à definição de conceitos e à construção de consensos relativos ao uso das Tecnologias de Informação e Comunicação (TICs) nas práticas de cuidados à saúde.

Dentre os conceitos mais importantes para compreender a aplicação das novas tecnologias em cuidados à saúde da população, pode-se destacar o termo "saúde digital”, que expressa todo o desenvolvimento do campo de uso de ciências da computação avançadas - nos campos de "big data", genômica e inteligência artificial, por exemplo - para fins relacionados à saúde, no sentido amplo. (WHO, 2017)

Outro conceito relevante é o de "saúde eletrônica" (eHealth ou eSaúde), que teve seu primeiro registro feito em 1897 no periódico The Lancet, para se referir a uma comunicação telefônica para diagnosticar uma criança com síndrome de Crupe. (FOSARELLI, 1983) Desde os anos 1990, algumas pesquisas mostram que os telefones móveis teriam crescente uso e aceitação como via para apoiar o cuidado da saúde, sendo utilizados em diversos contextos (LATTIMER et al., 1998; ODA; HEILBRON; TAYLOR, 1995) Em 2005, a OMS definiu e-Saúde como o uso seguro e econômico de TICs para dar suporte à saúde, incluindo tanto serviços de assistência e vigilância à saúde das pessoas, quanto atividades de educação em saúde, revisão de conhecimentos e pesquisa em saúde. (WHO, 2005) Em 2019, a OMS publicou um compendio com as recomendações sobre intervenções digitais para o fortalecimento do sistema de saúde. (WHO, 2019a)

No Brasil, o Ministério da Saúde (MS) publicou, em 2017, o documento “Estratégia e-saúde para o Brasil”, aprovado pela Resolução 
n 19 de 22 de junho de 2017, no qual declara que a e-Saúde tem como objetivo aumentar a qualidade e ampliar o acesso à atenção à saúde, de forma a qualificar as equipes de saúde, agilizar o atendimento e melhorar o fluxo de informações para apoio à decisão em saúde. Além disso, o documento descreve mecanismos contributivos para sua incorporação ao Sistema Único de Saúde (SUS) até 2020. (BRASIL, 2017a, 2017b)

Mais tarde, foi adotada pelo MS a terminologia "Saúde Digital" que é mais abrangente do que e-Saúde e incorpora os recentes avanços da tecnologia como novos conceitos, aplicações de redes sociais, internet das coisas, Inteligência Artificial (IA), entre outros. Com efeito, foi publicada a Resolução n ${ }^{\circ}$ 46, de 29 de agosto de 2019, que institui o Comitê Gestor da Estratégia de Saúde Digital e define a sua composição, as suas competências e as suas unidades operacionais na estrutura do MS, em substituição ao Comitê Gestor da Estratégia de e-Saúde no Brasil. (BRASIL, 2019a)

Além de Saúde Digital e e-Saúde, é importante apresentar as definições "Saúde Móvel” (mHealth), de "TS" e "TM".

A Saúde Móvel (mHealth, mSaúde) faz referência ao uso de tecnologias móveis, sem fio, para a saúde pública. (WHO, 2016) Apesar e ainda não existir uma definição padronizada para $m$ Health, ele vem sendo amplamente usado para nomear intervenções que se valem de dispositivos móveis, como telefones celulares e Assistentes Digitais Pessoais (PDAs), aparelhos de monitoramento de usuários nos serviços de saúde e outros dispositivos sem fios que constituem uma nova modalidade de assistência em saúde das pessoas. (WHO, 2011)

Segundo a American Telemedicine Association ${ }^{8}$ (ATA) (2006), o termo "Telessaúde" é frequentemente usado para definir a aplicação ampla de tecnologias eletrônicas de informação e comunicação

8 Associação Americana de Telemedicina. 
para realizar atividades de educação a distância e acompanhamento de pacientes pelos serviços de saúde. A TS usa as TICs para a troca de informações relativas a diagnóstico e tratamento de doenças e lesões, à pesquisa e avaliação e à educação continuada dos profissionais de saúde. (WHO, 2016)

No Brasil, é chamado de TS uma iniciativa com o objetivo de apoiar a organização de redes de atenção à saúde ordenadas pela Atenção Primária à Saúde (APS) no âmbito do SUS. Essa iniciativa envolve o desenvolvimento de ações de apoio à atenção à saúde e de educação permanente das equipes de APS, visando à educação para o trabalho, na perspectiva da melhoria da qualidade do atendimento, da ampliação do escopo de ações ofertadas por essas equipes, da mudança das práticas de atenção e da organização do processo de trabalho, por meio da oferta de teleconsultoria e telediagnóstico. (BRASIL, 2011)

Intimamente associado à TS está o termo "Telemedicina", que faz referência à prestação de cuidados médicos, em que a distância é um fator crítico. A TM usa as TICs para intercâmbio de informações, com o objetivo de apoiar o diagnóstico, o tratamento e a prevenção de doenças, a investigação e avaliação dos casos, e a educação continuada. (WHO, 2011) Esse intercâmbio pode ocorrer em tempo real (de forma síncrona), por exemplo, por telefone ou pelo uso de um link de vídeo ou pode ocorrer de forma assíncrona (armazenar e encaminhar), quando uma consulta é enviada e uma resposta fornecida posteriormente. O e-mail é um exemplo de intercâmbio assíncrono. (WHO, 2016) As primeiras especialidades médicas a incorporarem essas práticas em suas rotinas foram a radiologia, a dermatologia, a cardiologia e patologia. (AMERICAN TELEMEDICINE ASSOCIATION, 2006)

Na mesma linha, em 2002, o Conselho Federal de Medicina (CFM) do Brasil definiu a TM como o exercício da medicina mediado 
por tecnologias para fins de assistência, educação, pesquisa, prevenção de doenças e lesões e promoção de saúde. (CFM, 2019a)

Condicionadas à emergência em saúde pública, em caráter de excepcionalidade e enquanto durarem as medidas de enfrentamento ao coronavírus, o MS, em março de 2020, indicou que as ações de TM que envolvem interação a distância podem contemplar o atendimento pré-clínico, de suporte assistencial, de consulta, monitoramento e diagnóstico, no âmbito do SUS, bem como na saúde suplementar e privada. O atendimento deverá ser efetuado diretamente entre médicos e pacientes, por meio de TICs que garanta a integridade, a segurança e o sigilo das informações. (KARPOV, 2020)

Para nomear a relação mediada por TICs que põe em contato direto profissionais de saúde e pacientes - ou profissionais entre si, envolvidos com pacientes -, se reserva o termo "Telessaúde". Nos casos em que o profissional é o médico, adota-se o termo “Telemedicina”. As ações desenvolvidas entre os profissionais de saúde e os usuários de serviços de saúde englobam a teleconsultoria, o telediagnóstico, a teleducação, a teleinterconsulta, o telemonitoramento e a teleorientação, cujas definições são apresentadas no quadro a seguir. 
Quadro 1 - Termos e definições utilizados relacionados à TS

\begin{tabular}{|c|c|c|}
\hline Termo & Definição & Fonte \\
\hline Teleconsultoria & $\begin{array}{l}\text { Consultoria registrada e realizada entre } \\
\text { trabalhadores, profissionais e gestores da } \\
\text { área de saúde, por meio de instrumentos de } \\
\text { telecomunicação bidirecional, com o propósito de } \\
\text { esclarecer dúvidas sobre procedimentos clínicos, } \\
\text { ações de saúde e questões relativas ao processo de } \\
\text { trabalho em saúde, podendo ser em tempo real ou } \\
\text { por meio de mensagens off-line }\end{array}$ & $\begin{array}{l}\text { M.S } 2011 \\
\text { Portaria } \\
\mathrm{n}^{\circ} 2.546\end{array}$ \\
\hline Telediagnóstico & $\begin{array}{l}\text { Consiste em serviço autônomo que utiliza as } \\
\text { TICs para a realização de serviços de Apoio ao } \\
\text { Diagnóstico, como a avaliação de exames à distância, } \\
\text { facilitando o acesso a serviços especializados. Busca } \\
\text { reduzir o tempo de diagnóstico possibilitando } \\
\text { tratamento para complicações previsíveis por meio } \\
\text { do diagnóstico precoce. }\end{array}$ & $\begin{array}{l}\text { M.S } 2011 \\
\text { Portaria } \\
\mathrm{n}^{\circ} 2.546\end{array}$ \\
\hline Teleducação & $\begin{array}{l}\text { Disponibilização de objetos de aprendizagem } \\
\text { interativos sobre temas relacionados à saúde, } \\
\text { ministrados a distância por meio de TICs, com foco } \\
\text { na aprendizagem no trabalho. }\end{array}$ & $\begin{array}{l}\text { M.S } 2011 \\
\text { Portaria } \\
\mathrm{n}^{\circ} 2.546\end{array}$ \\
\hline Teleinterconsulta & $\begin{array}{c}\text { Exclusivamente para troca de informações e } \\
\text { opiniões entre médicos, para auxílio diagnóstico ou } \\
\text { terapêutico. }\end{array}$ & $\begin{array}{c}\text { Ofício CFM n } \\
1756 / 2020\end{array}$ \\
\hline Telemonitoramento & $\begin{array}{l}\text { Monitoramento a distância de parâmetros de saúde } \\
\text { e/ou doença de pacientes por meio das TICs, que } \\
\text { pode incluir a coleta de dados clínicos. }\end{array}$ & $\begin{array}{l}\text { M.S } 2011 \\
\text { Portaria } \\
\mathrm{n}^{\circ} 2.546\end{array}$ \\
\hline Teleorientação & $\begin{array}{l}\text { Indicado para que profissionais da medicina } \\
\text { realizem a distância a orientação e o } \\
\text { encaminhamento de pacientes em isolamento. }\end{array}$ & $\begin{array}{c}\text { Ofício CFM n } \\
1756 / 2020\end{array}$ \\
\hline
\end{tabular}

Fonte: elaborado pelos autores. 


\section{Aplicações da TS durante \\ a pandemia de COVID-19}

Os estudos analisados evidenciaram diversas possibilidades de aplicação da telessaúde durante a pandemia de Covid-19, desde na atenção à saúde de pacientes, através de teleconsultas, por exemplo; até no uso dessa estratégia para a capacitação profissional e para o apoio psicológico a profissionais de saúde.

\section{Atenção a pacientes}

Diversos países no mundo têm utilizado os recursos da TS em suas estratégias de combate à pandemia. Muitos estudos reportam a utilização de videochamadas para realizar triagem para pacientes com suspeita de infecção pelo coronavírus. (CFM, 2019b; DING et al., 2020; GONG et al., 2020; PAPADIMOS et al., 2020; PORTNOY; WALLER; ELLIOTT, 2020; HONG et al., 2020; NORRIS, 2002; SONG; LIU; WANG, 2020; SUN et al., 2020) Essa triagem inclui a avaliação de sintomas e possíveis sinais de insuficiência respiratória aguda. (NORRIS, 2002)

Alguns hospitais têm utilizado a TS para realizar a triagem de pacientes com sintomas de infecção do trato respiratório em condição estável, seja através de chamada telefônica ou videoconferência (PEREZ-ALBA et al., 2020), seja através de um formulário do Google Forms, compartilhado sob a forma de código de resposta rápida para a realização da primeira avaliação dos pacientes sem contato face a face. (PEREZ-SUST et al., 2020) Assim, a avaliação presencial é indicada apenas para casos selecionados, a partir da triagem não presencial.

O telemonitoramento também vem sendo utilizado, especialmente por profissionais médicos que aproveitam os recursos dos prontuários eletrônicos para acesso remoto. (PALADINO et al., 2017; PAPADIMOS, 2020) Essa estratégia tem sido utilizada tanto em enfermarias quanto em UTIs, permitindo que enfermeiros e médicos 
acompanhem remotamente pacientes críticos em diversos hospitais. (JOHN, 2020)

Na China, serviços de TS ou TM vêm sendo chamados de "hospitais virtuais" e oferecem apoio a unidades de saúde por meio de teleconsultoria, evitando deslocamentos de pacientes que possam causar infecções cruzadas. O Hospital da Universidade de Sichuan lançou um sistema de TM em vídeo em tempo real, por meio do qual uma equipe multidisciplinar provê consultas para pessoas com COVID-19, concentrando-se na atenção a grupos vulneráveis e sintomas graves da doença. (ZHEN, 2020) Ademais, para pacientes com condições crônicas, os hospitais virtuais oferecem acompanhamento regular, instruções sobre uso de medicamentos e entrega de medicamentos sem contato. (KESHVARDOOST; BAHAADINBEIGY; FATEHI, 2020; HOLLANDER, 2020)

Estudos realizados na Itália (GHOSH; GUPTA; MISRA, 2020), Estados Unidos da América (EUA) (SUN et al., 2020; HOLLANDER; CARR, 2020) e China (KUMMITHA, 2020; SUN et al., 2020) relatam o desenvolvimento de aplicativos para smartphone usados para identificação de casos e rastreamento de contatos. Também são realizadas consultas on-line gratuitas e intervenções psicológicas. (ZHEN, 2020)

A triagem, o monitoramento e a integração do atendimento virtual são aplicações de uma plataforma digital de gerenciamento de crises, desenvolvida no Canadá com o objetivo de melhorar a resposta do sistema de saúde com base nas evidências científicas sobre a COVID-19. A plataforma centraliza os dados do processo de triagem, acompanhamento de pacientes, atendimento virtual integrado. (KRAUSZ et al., 2020)

A aplicação da TS não se restringiu aos países desenvolvidos. No Afeganistão, por exemplo, organizou-se uma pequena rede de consultas usando aplicativos móveis para oferecer orientações de 
saúde, prevenindo exposições desnecessárias de pacientes e profissionais de saúde. (AZIZY; FAYAZ; AGIRBASLI, 2020)

No Brasil, o MS tem feito o monitoramento a distância da saúde da população por meio do TeleSUS. Os indivíduos têm recebido, desde o mês de abril, chamadas telefônicas - através do número 136 - que buscam identificar antecipadamente pessoas com sinais e sintomas de infecção por coronavírus. (BRASIL, 2020a)

Outro exemplo de uso da TS no Brasil é o aplicativo Coronavírus-SUS. O aplicativo disponibiliza informações diversas sobre a COVID-19 como os sintomas e as formas de prevenção. A pessoa que apresenta sintomas pode conferir se são compatíveis com os da doença e, caso sejam, será instruído e encaminhado à unidade básica de saúde mais próxima. (BRASIL, 2020a) O aplicativo permite ainda que a Secretaria de Vigilância em Saúde, do MS divulgue comunicados aos usuários em geral ou a públicos específicos.

Um terceiro exemplo refere-se ao Tele-Coronavírus 155, fruto de uma parceria entre o Governo da Bahia, a Universidade Federal da Bahia (UFBA) e a Fundação Oswaldo Cruz (Fiocruz). Voltado para orientar o público em geral, esse serviço visa reduzir a procura de serviços de saúde por pessoas assintomáticas ou com sintomas leves, diminuindo o risco de contágio. (BAHIA, 2020) O serviço é gratuito, está disponível das 7 h̀ às 19h, e conta com a participação de mais de 1.200 estudantes de cursos de Medicina, voluntários, prestando orientação à população, sob supervisão de médicos. Até o dia $1^{\circ}$ de agosto, já tinham sido feitos mais de 110 mil atendimentos via chamada telefônica.

\section{Capacitação de profissionais de saúde}

Ações de educação a distância - Teleducação - sobre COVID-19 e outros temas na área da saúde têm sido desenvolvidas através de web aulas, web palestras, webinars, podcasts, cursos on-line e até mesmo lives em redes sociais, sendo ofertadas tanto para profissionais que estão 
na linha de frente do atendimento a pacientes, quanto para outros profissionais e para estudantes (residentes, internos, estagiários).

Recursos de e-learning, a exemplo das plataformas Zoom (Zoom Video Communications Inc, San Jose, CA, USA) e Microsoft Teams (Microsoft Corp, Redmond, WA, EUA) têm sido utilizados com frequência pelos serviços de saúde para educação permanente de pessoal, com realização de reuniões, discussões de casos, compartilhamento de material didático, dentre outros.

Dentre as atividades possíveis com o uso desses recursos no apoio à formação médica, podem-se citar: discussões de casos por meio de clínicas virtuais com acesso a informações de prontuários, encontros clínicos e feedback dos professores via conferências virtuais (MIRANDA et al., 2020); visitas supervisionadas através de telefone ou vídeo (PLOCIENNICZAK et al., 2020); realização de sessões clínicas, seminários on-line, clubes de periódicos e disponibilização de palestras por meio de podcasts. (LOH; HSIAO; SHI, 2020)

A TS tem sido bastante utilizada para a oferta de cursos de capacitação e educação permanente de profissionais da saúde por meio de plataformas gerenciadas pela OMS e, no Brasil, pela Universidade Aberta do SUS (Unasus), como se pode ver no Quadro 2. 
Quadro 2 - Capacitações e aperfeiçoamento sobre COVID-199

\begin{tabular}{|c|c|c|}
\hline Descrição & $\begin{array}{l}\text { Órgão/Instituição } \\
\text { responsável }\end{array}$ & Link \\
\hline $\begin{array}{l}\text { Página com links de vídeos informativos } \\
\text { sobre: manejo clínico e tratamento na } \\
\text { Atenção Básica e Atenção Especializada; } \\
\text { cursos em parceria com a UNA-SUS; } \\
\text { saúde da pessoa com deficiência, saúde } \\
\text { mental, saúde indígena, orientações } \\
\text { gerais e outros. }\end{array}$ & Ministério da Saúde & $\begin{array}{l}\text { https://coronavirus.saude. } \\
\text { gov.br/capacitacao }\end{array}$ \\
\hline $\begin{array}{l}\text { Cursos sobre COVID-19 voltado para } \\
\text { profissionais de saúde pública, gerentes } \\
\text { de incidentes e pessoas que trabalham } \\
\text { para as Nações Unidas, organizações } \\
\text { internacionais e ONGs. }\end{array}$ & $\begin{array}{l}\text { Organização Mundial } \\
\text { da Saúde (OMS/WHO) }\end{array}$ & $\begin{array}{l}\text { https://openwho.org/ } \\
\text { courses }\end{array}$ \\
\hline $\begin{array}{l}\text { Curso sobre manejo da infecção } \\
\text { causada pelo novo coronavírus. }\end{array}$ & Fiocruz & $\begin{array}{c}\text { https://campusvirtual. } \\
\text { fiocruz.br/gestordecursos/ } \\
\text { hotsite/covid19 }\end{array}$ \\
\hline $\begin{array}{l}\text { Cursos sobre coronavírus } \\
\text { com sessões destinadas a orientações } \\
\text { para profissionais de saúde e para } \\
\text { população geral. }\end{array}$ & $\begin{array}{l}\text { Universidade Aberta } \\
\text { do SUS (UNASUS) }\end{array}$ & $\begin{array}{l}\text { https://www.unasus.gov.br/ } \\
\text { especial/covid19/ }\end{array}$ \\
\hline $\begin{array}{l}\text { COVID-19 Atualização e evidências } \\
\text { para profissionais da saúde, curso } \\
\text { autoinstrucional e gratuito } \\
\text { e link para inscrição. }\end{array}$ & $\begin{array}{c}\text { Faculdade de Medicina } \\
\text { da Universidade de São } \\
\text { Paulo (FMUSP) }\end{array}$ & $\begin{array}{l}\text { https://eephcfmusp.org. } \\
\text { br/portal/online/curso/ } \\
\text { curso-covid-19/ }\end{array}$ \\
\hline
\end{tabular}

Fonte: elaborado pelos autores.

9 É importante destacar que existem no Brasil diferentes iniciativas de capacitação aos profissionais e estudantes da área da saúde, realizadas por universidades, prefeituras, iniciativa privada e outros. No entanto, visto a pluralidade de oferta, a dificuldade temporal de sistematização coerente de tais cursos de capacitação para o presente capítulo, adotou-se a seleção das principais capacitações disponíveis a profissionais de saúde no atual contexto. 


\section{Apoio psicológico a profissionais de saúde}

A experiência de enfrentamento à pandemia, nos diversos níveis da atenção à saúde, tem afetado diretamente o bem-estar e a saúde mental dos profissionais envolvidos. O percentual de trabalhadores da saúde afetados pela COVID-19, em todo o mundo, varia entre $8 \%$ e $10 \%$. (FUNDACENTRO, 2020) O potencial de contaminação aumentado diante da constante exposição ao vírus, associado às condições de trabalho estressantes, tem colocado os profissionais diante de inúmeras questões relativas à sua própria segurança bem como a de seus familiares. (CHEN et al., 2020)

Além disso, dilemas éticos relativos à tomada de decisão sobre condutas clínicas em unidades de saúde superlotadas, quando não se pode oferecer a todos os cuidados que cada um necessita, têm afetado de forma direta a saúde mental dos profissionais. (ARMITAGE; NELLUMS, 2020; MENON; PADHY, 2020) De modo semelhante, o convívio diário com a morte de pacientes e colegas de trabalho e a fragilização dos vínculos sociais com familiares e amigos, devido ao isolamento social, evocam processos emocionais e comportamentais complexos. (ARMITAGE; NELLUMS, 2020; KANG et al., 2020; MASSEY et al., 2020) Tudo isso tem contribuído para o surgimento de sintomas de estresse pós-traumático, sintomas depressivos, distúrbios do sono, ansiedade, medo, desamparo, raiva e outras manifestações com efeitos importantes sobre a saúde mental a curto e longo prazo.

Tal cenário tem demonstrado a existência de lacunas e falhas no preparo psicológico das equipes de saúde para o enfrentamento de situações de crises de proporção generalizada, como o de uma pandemia. Consequentemente, tem-se observado um esforço por parte de órgãos governamentais e de universidades para a realização de pesquisas que identifiquem as melhores estratégias de proteção da saúde mental dos profissionais de saúde da linha de frente do combate à COVID-19. 
Para promover o cuidado em saúde mental a esses profissionais, a TS tem sido muito utilizada. No plano internacional, algumas experiências se destacam, em Wuhan, China, profissionais de saúde de língua chinesa dos EUA, Canadá e Austrália criaram através de um aplicativo de mídias sociais e mensagens um grupo de acolhimento aos profissionais da linha de frente permitindo vínculo, compartilhamento de experiências e escuta de modo coletivo e individual. (CHENG et al., 2020) Em Nova York, EUA, foram utilizadas estratégias com grupos de apoio virtual facilitados por assistentes sociais e psicólogos. (RIPP; PECCORALO; CHARNEY, 2020) Na China, no Brasil e nos Estados Unidos, também foram disponibilizadas linhas diretas de telefone para o gerenciamento imediato de crises, onde os profissionais podem entrar em contato e assim lidar com seus problemas no momento em que estes estão mais intensos. (KANG et al., 2020; FUKUTI et al., 2020; RIPP; PECCORALO; CHARNEY, 2020; YAO et al., 2020)

No Brasil, o MS abriu um canal de teleconsulta psicológica para os trabalhadores de saúde - o TeleSUS -, no qual o suporte é oferecido por videochamada, utilizando estratégias de manejo de crise para escuta e acolhimento desses profissionais. (KARPOV, 2020) A partir desse contato, os profissionais identificados com risco ou sintomatologia muito intensos são encaminhados para avaliação psiquiátrica ou outro dispositivo de suporte. E a Faculdade de Medicina da Universidade de São Paulo e seu Complexo de Saúde, o Hospital das Clínicas, desenvolveram o Programa "COMVC19: Equipamentos de Proteção Individual para a Saúde Mental e o Bem-Estar Psicossocial aos Profissionais de Saúde no Combate ao Pandemia do COVID-19”, o qual prevê apoio psicossocial e de saúde mental a aproximadamente 20 mil funcionários hospitalares. (FUKUTI et al., 2020) 


\section{Reorganização dos serviços de saúde}

Além das diversas possibilidades do uso da telessaúde relacionadas à assistência aos pacientes e ao apoio profissional, este recurso vem sendo utilizado largamente na organização dos serviços de saúde em diferentes países no mundo.

Atenção às doenças crônicas

e oferta de cuidados continuados

Em função das medidas de distanciamento social implementadas com o objetivo de diminuir a transmissão da COVID-19, os serviços de saúde vêm passando por modificações significativas no que tange à atenção às doenças crônicas ou à oferta de cuidados contínuos, como na assistência em obstetrícia e à saúde sexual e prevenção ao Vírus da Imunodeficiência Humana (HIV). Em que pese a importância dessas medidas, os serviços de saúde precisam estar atentos à necessidade de continuidade de tratamentos, a fim de manter ou atingir o controle da condição crônica e evitar a aquisição de novas doenças, o que inclui a prevenção da COVID-19.

Assim, a pandemia de COVID-19 tem impulsionado a reorganização dos sistemas de saúde de modo a assegurar a assistência à saúde a sujeitos acometidos pelo novo coronavírus, sem descuidar dos indivíduos com doenças crônicas e outras necessidades de saúde.

Nesse cenário, muitos estudos e relatos apontam o uso da TS e da TM como alternativa com resultados promissores. Considerando os benefícios dessas novas modalidades de oferta de cuidados em saúde em um conjunto de doenças e agravos, espera-se que a incorporação dessas tecnologias se difunda ainda mais a médio e longo prazo.

Em todo o mundo, tem crescido a utilização da TS por profissionais de diversas especialidades para o atendimento a pessoas com 
condições de saúde não urgentes e/ou de acompanhamento de pessoas com doenças crônicas ou que necessitam de cuidados contínuos.

No Quadro 3, é possível observar várias aplicações da TS, desenvolvidas para manter a assistência a diferentes grupos etários, como crianças e idosos, e com distintos diagnósticos clínicos, como diabetes, neoplasias, doenças cardiovasculares. Esses achados tornam evidentes as múltiplas possibilidades do atendimento e acompanhamento remoto na atenção à saúde da população durante períodos de pandemia.

Quadro 3 - Síntese com aplicações da TS para cuidados contínuos à saúde

\begin{tabular}{|c|c|c|}
\hline $\begin{array}{l}\text { Aplicações da } \\
\text { Telessaúde }\end{array}$ & Descrição & Referência \\
\hline $\begin{array}{l}\text { Gravidez } \\
\text { e Puerpério }\end{array}$ & $\begin{array}{l}\text { Obstetrícia com interação através } \\
\text { de páginas em redes sociais, telefones } \\
\text { celulares ou tablets; serviço de pré-natal } \\
\text { baseado em telemedicina com envio de } \\
\text { materiais educacionais via e-mail; visitas } \\
\text { virtuais com um nutricionista e um aplicativo } \\
\text { de telefone para documentar valores de } \\
\text { glicose no sangue. }\end{array}$ & $\begin{array}{l}\text { ALSHARAYDEH et al. } \\
\text { (2020) } \\
\text { PEAHI; SMITH; } \\
\text { MONIZ (2020) } \\
\text { AZIZ et al., 2020 }\end{array}$ \\
\hline Pediatria & $\begin{array}{l}\text { Consultas virtuais para controle de peso } \\
\text { para crianças com obesidade, uso de } \\
\text { dispositivos de monitoramento remoto } \\
\text { e compartilhamento virtual de materiais } \\
\text { educativos; consultas virtuais e linha direta } \\
\text { de apoio para avaliar o controle de asma; } \\
\text { atendimentos em cardiologia; apoio } \\
\text { psicossocial em saúde mental } \\
\text { e uso de educação interativa remota } \\
\text { como estratégia de psicoeducação. }\end{array}$ & $\begin{array}{c}\text { BADAWY; RADOVIC } \\
(2020) \\
\text { BARNEY et al. } \\
(2020) \\
\text { O'HARA; } \\
\text { JOHNSTON; } \\
\text { BROWNE (2020) } \\
\text { PAPADOPOULOS } \\
\text { et al. (2020) } \\
\text { CHOWDHURY et al. } \\
(2020) \\
\text { YE (2020) }\end{array}$ \\
\hline
\end{tabular}




\begin{tabular}{|c|c|c|}
\hline $\begin{array}{l}\text { Aplicações da } \\
\text { Telessaúde }\end{array}$ & Descrição & Referência \\
\hline HIV/AIDS & $\begin{array}{l}\text { Plataformas on-line e de comunicação virtual } \\
\text { como instrumentos de apoio à prevenção } \\
\text { da COVID-19 e a continuidade dos serviços } \\
\text { especializados para manter a adesão ao } \\
\text { tratamento do HIV e o envolvimento com os } \\
\text { cuidados preventivos. Oferta de atendimento, } \\
\text { que inclui apoio social e mental, além da } \\
\text { entrega de preservativos, autoteste para HIV e } \\
\text { medicamentos como PREP em domicílio. }\end{array}$ & $\begin{array}{l}\text { ADEPOJU et al. } \\
(2020) \\
\text { DOURADO et al. } \\
(2020) \\
\text { HOAGLAND et al. } \\
(2020)\end{array}$ \\
\hline Diabetes & $\begin{array}{l}\text { Consultas convertidas em telemedicina com } \\
\text { envio de fotos para acompanhamento clínico } \\
\text { em casos de ulcerações; dados de medidor/ } \\
\text { monitor de glicose repassados para um } \\
\text { computador ou smartphone; informações } \\
\text { sobre glicemia, pressão arterial, peso corporal } \\
\text { e número de passos diários automatizados e } \\
\text { transferidos } \\
\text { via smartphone. }\end{array}$ & $\begin{array}{l}\text { AHN (2020) } \\
\text { MELONI et al. } \\
(2020)\end{array}$ \\
\hline $\begin{array}{c}\text { Doenças } \\
\text { Cardiovasculares }\end{array}$ & $\begin{array}{l}\text { Teleconsultas com exames virtuais e planos } \\
\text { de atendimento multiprofissional; dados } \\
\text { de prontuário eletrônico, obtenção de sinais } \\
\text { vitais e traçados de Eletrocardiograma (ECG) } \\
\text { a partir de wearables digitais; monitoramento } \\
\text { de taxa e ritmo através de aplicativo de celular } \\
\text { para o tratamento de Fibrilação Atrial (FA); } \\
\text { emissão de prescrições eletrônicas; inspeções } \\
\text { no local da incisão após implante de } \\
\text { dispositivos cardíacos ou ablação do cateter; } \\
\text { para manejo de doenças valvares e estruturais, } \\
\text { com transmissão de dados de imagem e } \\
\text { vigilância telefônica para pacientes graves e } \\
\text { outros recursos remotos como a transição } \\
\text { para programas de reabilitação cardíaca } \\
\text { on-line para apoiar as pessoas que vivem com } \\
\text { Doença Cardiovascular (DCV). }\end{array}$ & $\begin{array}{l}\text { BRYANT; FEDSON; } \\
\text { SHARAFKHANEH; } \\
\text { (2020) } \\
\text { LAKKIREDDY, et al. } \\
(2020) \\
\text { PLUYMAEKERS } \\
\text { et al., (2020) } \\
\text { PASKUDZKA et al. } \\
\text { (2020) } \\
\text { PLOŃSKA- } \\
\text { GOŚCINIAK et al. } \\
\text { (2020) } \\
\text { NEUBECK et al. } \\
\text { (2020) }\end{array}$ \\
\hline
\end{tabular}




\begin{tabular}{|c|c|c|}
\hline $\begin{array}{l}\text { Aplicações da } \\
\text { Telessaúde }\end{array}$ & Descrição & Referência \\
\hline $\begin{array}{l}\text { Doenças } \\
\text { Respiratórias } \\
\text { Crônicas }\end{array}$ & $\begin{array}{l}\text { Teleconsultas com pneumologista } \\
\text { e fisioterapeuta para reabilitação pulmonar. }\end{array}$ & $\begin{array}{c}\text { BRYANT; FEDSON; } \\
\text { SHARAFKHANEH } \\
(2020)\end{array}$ \\
\hline $\begin{array}{c}\text { Doenças do } \\
\text { Aparelho Digestivo }\end{array}$ & $\begin{array}{l}\text { Acompanhamento ambulatorial via telessaúde, } \\
\text { com discussões multidisciplinares on-line e } \\
\text { entrega de medicamentos em domicílio; uso } \\
\text { de aplicativos e implementação digital } \\
\text { de abordagens e algoritmos baseados em } \\
\text { evidências para doença inflamatória intestinal. }\end{array}$ & $\begin{array}{l}\text { ALLOCCA et al. } \\
(2020) \\
\text { LEES; REGUEIRO; } \\
\text { MAHADEVAN } \\
(2020)\end{array}$ \\
\hline Neoplasias & $\begin{array}{l}\text { Consultas via telemedicina para pacientes } \\
\text { internados e ambulatoriais, com visitas } \\
\text { a subespecialidades, gerenciamento de } \\
\text { comorbidades e consultas de segunda opinião. } \\
\text { Como ferramenta para rastrear pacientes } \\
\text { que precisam de visitas de acompanhamento } \\
\text { pessoalmente e como instrumento facilitador } \\
\text { para a comunicação entre diferentes } \\
\text { especialistas, favorecendo uma abordagem } \\
\text { multidisciplinar. Oferta de serviços clínicos } \\
\text { oncológicos via telemedicina para locais } \\
\text { remotos como comunidades rurais e sistema } \\
\text { penitenciário. Telessaúde como um meio } \\
\text { eficaz para oferecer atendimento } \\
\text { domiciliar a idosos com câncer. }\end{array}$ & $\begin{array}{l}\text { LEWIS et al., (2020) } \\
\text { ELKADDOUM et al. } \\
\text { (2020) } \\
\text { NGUYEN et al. } \\
\text { (2020) }\end{array}$ \\
\hline $\begin{array}{l}\text { Transtornos } \\
\text { Mentais }\end{array}$ & $\begin{array}{l}\text { Prestação de serviços de psiquiatria via } \\
\text { telessaúde; assistência remota para pessoas } \\
\text { com transtornos mentais graves, que inclui } \\
\text { o acesso aos medicamentos necessários e à } \\
\text { equipe de assistência em saúde mental. }\end{array}$ & $\begin{array}{l}\text { LOOI; PRING (2020) } \\
\text { BARTELS et al. } \\
(2020) \\
\text { KHATRI; PERRONE } \\
(2020)\end{array}$ \\
\hline
\end{tabular}




\begin{tabular}{|c|c|c|}
\hline $\begin{array}{l}\text { Aplicações da } \\
\text { Telessaúde }\end{array}$ & Descrição & Referência \\
\hline Saúde do Idoso & $\begin{array}{c}\text { Assistência social e de saúde por televisão e } \\
\text { telefone, TV-AssistDem (serviço integrado } \\
\text { assistido baseado na TeleVision para apoiar } \\
\text { adultos europeus que vivem com demência } \\
\text { leve ou comprometimento cognitivo } \\
\text { leve). Estímulo à conexão social durante } \\
\text { o distanciamento físico através de tablets, } \\
\text { computadores e celulares. Avaliação geriátrica } \\
\text { pré-consulta realizada por telefone } \\
\text { aos pacientes (idosos frágeis) }\end{array}$ & $\begin{array}{c}\text { GOODMAN- } \\
\text { CASANOV, et al. } \\
(2020) \\
\text { EDELMAN et al. } \\
(2020) \\
\text { DIGIOVANNI et al. } \\
(2020)\end{array}$ \\
\hline
\end{tabular}

Fonte: elaborado pelos autores.

\section{Atenção a populações específicas \\ (indígenas, quilombolas, entre outros)}

Os desafios enfrentados pelos serviços de saúde a fim de garantir o acesso a populações que vivem em zonas rurais, indígenas e quilombolas têm sido ainda mais complexos. São populações que têm sido historicamente vulnerabilizadas nos diversos aspectos da vida em sociedade: educação, renda, habitação, saneamento, emprego, dentre outros. Essas desigualdades se expressam também na saúde. (CAETANO et al., 2020; SINGH; SIAHPUSH, 2014)

De fato, estudos apontam que comunidades rurais, indígenas e quilombolas, em geral, têm menor acesso a ações e serviços públicos de saúde em comparação com as populações urbanas (LADITKA; LADITKA; PROBST, 2009; PROBST et al., 2004), o que acrescenta mais um desafio ao enfrentamento da COVID-19 nessas populações. (HIRKO et al., 2020) 
Nesse contexto, o uso da TS pode trazer benefícios, como a diminuição de tempo para que o atendimento seja iniciado, a redução de custos de deslocamento de pacientes e profissionais de saúde e a melhoria da qualidade assistencial, ao possibilitar a interconsulta entre especialistas e generalistas atuantes em áreas rurais ou isoladas. (BRADFORD; CAFFERY; SMITH, 2016; CAFFERY, 2016; DU TOIT et al., 2019)

Uma análise de custo-consequência com indígenas em uma comunidade remota, em Queensland, na Austrália, demonstrou que a oferta do serviço por TS custou AU\$284 (dólares australianos), enquanto levar a equipe até a área custou AU\$482 e pagar a passagem do usuário até a clínica na cidade e ofertar o serviço custou no total AU\$692, demonstrando que o serviço de TS teve menor custo. (SNOSWELL et al., 2019) Saliente-se que esse estudo analisou apenas o custo da ofertar das consultas, não avaliando a qualidade da atenção ofertada em cada modalidade.

Portanto, apesar da TS poder se configurar como uma importante ferramenta para diminuir a desigualdade no acesso às ações e serviços de saúde de populações vulneráveis em área rurais, indígenas ou quilombolas, ainda há questionamentos sobre a qualidade da atenção ofertada nessa modalidade. (ANDERSON et al., 2017; ASHFAQ et al., 2020; ROMANICK-SCHMIEDL; RAGHU, 2020)

Esse fato leva a outro questionamento ético e político, relativo à decisão de ofertar serviços menos custosos e potencialmente de menor qualidade para populações que historicamente têm o direito à saúde relativizado e dificultado por condições políticas e estruturais. Nesse caso, o questionamento pode ser respondido pela garantia da qualidade da atenção ofertada por meio da TS. Para tal, é necessário investir no desenvolvimento da TS, tanto em seus aspectos tecnológicos, quanto em relação à participação de profissionais e usuários na definição das formas de sua utilização. 
Com efeito, além da análise de custo e efetividade, é fundamental assegurar espaços de participação dessas populações, promovendo a tomada de decisão compartilhada sobre o modelo de atenção ofertado e sobre todas as políticas públicas que lhes dizem respeito.

\section{Desafios à utilização da telessaúde}

Apesar da potencialidade e de todas as vantagens da aplicação da TS, há também desafios para o desenvolvimento de ações de cuidado à saúde com base nas TICs.

Em primeiro lugar, há o desafio da adaptação dos profissionais a novas tecnologias, o que incluiu a integração com novas plataformas digitais. (MANN et al., 2020; PÉREZ-SUST, 2020) O desconhecimento sobre o tema ainda é presente entre médicos (AYATOLLAHI; SARABI; LANGARIZADEH, 2015; KIBERU; SCOTT; MARS, 2019) e a adoção da teleconsulta ainda é baixa, como demonstrou uma pesquisa feita em 2017 no Canadá, em que apenas $24 \%$ dos médicos tiveram alguma experiência no cuidado de pacientes com HIV. (ANDERSON et al., 2017) Superar esse obstáculo requer um esforço inicial de aprendizado do uso da TS, que precisa ser ainda maior em locais onde não é ofertado um suporte tecnológico robusto e bem integrado à infraestrutura de saúde de modo a atender rapidamente às dúvidas e demandas dos profissionais de saúde. (AZIZY; FAYAZ; AGIRBASLI, 2020; HUMPHREYS et al., 2020; MANN et al., 2020)

Um segundo desafio se refere ao desconhecimento ou à indisponibilidade da TS para os usuários. Com efeito, a TS e mesmo a TM não são de amplo conhecimento dos usuários dos serviços de saúde. Em 2019, pesquisa realizada nos EUA demonstrou que $29 \%$ dos pacientes que não usaram TM acreditavam que esses serviços 
não estavam disponíveis para eles e 37\% não sabiam se a TM era oferecida. (POWER, 2019) Esses achados apontam falhas no processo de comunicação entre os prestadores de serviços de saúde e os seus usuários no que se refere às ações ofertadas na modalidade não presencial.

Mesmo quando se tem conhecimento sobre a oferta de serviços mediados por TIC, alguns pacientes não têm acesso ou familiaridade com computadores, smartphones ou internet de alta velocidade e esse tem sido mais um grande desafio para a implementação da TS no mundo. (AZIZY; FAYAZ; AGIRBASLI, 2020; CALTON; ABEDINI; FRATKIN, 2020; KESHVARDOOST; BAHAADINBEIGY; FATEHI, 2020; OMBONI, 2020)

Além do desconhecimento sobre a disponibilidade de serviços de TS, há a barreira cultural que pode se impor como um quarto desafio para a implantação dessa tecnologia nos serviços. (KIBERU; SCOTT; MARS, 2019) O uso da TS pode ser assustador para alguns pacientes, como relatado em um estudo na Índia (PAPADIMOS et al., 2020) e em um estudo com veteranos nos EUA (ANDREWS et al., 2017), especialmente no grupo de idosos e sem instrução formal, com pouco conhecimento técnico para utilizar as plataformas de consulta. Nesse contexto, vale ressaltar que enfrentar esse desafio é importante, sobretudo, visando à população idosa que é potencialmente uma das maiores beneficiárias da TS, haja vista a necessidade de cuidados contínuos de condições crônicas que costumam ser muito prevalentes nessa faixa etária.

Um quinto desafio ao uso da TS se relaciona à infraestrutura tecnológica. Tanto os usuários precisam ter equipamentos e softwares que suportem consultas de vídeo de alta qualidade, quanto hospitais e instituições precisam de grande capacidade tecnológica para atender a todas as necessidades de transferência de dados. (POWER, 2019) Sem uma infraestrutura adequada, a TS pode gerar frustração nos profissionais. (SHAH et al., 2013; 
VIDAL-ALABALL et al., 2020) Com efeito, um estudo (WHITTEN; ADAMS, 2003) mostrou que a TM foi mais bem avaliada nos locais com melhor estrutura física e logística, maior conhecimento tanto de profissionais, gestores e usuários - e melhor resposta do sistema de suporte aos problemas de usabilidade da TS. Para enfrentar esse desafio, é fundamental treinar as equipes no uso das tecnologias, ouvindo as demandas dos profissionais de modo a melhorar a usabilidade dos softwares envolvidos na atenção à saúde ofertada.

Outro desafio significativo se refere à heterogeneidade das ferramentas disponíveis que, aliada à falta de um repositório comum para todas as informações dos pacientes, pode impedir a obtenção do máximo benefício dessas soluções digitais. (OMBONI, 2020; SMITH; THOMAS; SNOSWELL et al., 2020) Nesse ponto, a questão da interoperabilidade é essencial.

Um sétimo desafio é o receio de médicos de que a TS afaste os usuários dos serviços presenciais, principalmente, na atenção primária, comprometendo a criação ou a manutenção de vínculo entre profissional e usuários e dificultando a realização de avaliações mais abrangentes da condição de saúde do usuário. (ANDERSON et al., 2017; ASHFAQ et al, 2020) De fato, é possível que a consulta via TS dificulte a percepção pelos profissionais de problemas que mesmo na consulta tradicional, face a face, já exigem um alto nível de escuta e habilidade em relações interpessoais. (ROMANICK-SCHMIEDL; RAGHU, 2020)

Outro desafio bastante discutido se atém à privacidade, à segurança dos dados e a outras questões de ética digital. (OMBONI, 2020; RAHMAN et al., 2020) Essas questões assumem grande vulto, quando se sabe que a informação coletada da vida cotidiana das pessoas tem se tornado um dos mais valiosos ativos econômicos do mundo contemporâneo, seja do ponto de vista financeiro, seja do ponto de vista político. Diversas empresas têm interesse 
econômico em acessar grandes bases de dados com informações sobre a saúde das pessoas, o que impõe a necessidade de séria regulamentação pelos países para garantir a efetiva proteção de informações e manter a privacidade dos dados dos indivíduos que usam os serviços de TS. Essas iniciativas de segurança precisam ser acompanhadas de legislação específica para evitar fraudes por parte de instituições que não respeitem princípios éticos e legais acordados pela sociedade em cada nação.

Por último, há o desafio da cobertura de internet. Segundo o Centro Regional de Estudos para o Desenvolvimento da Sociedade da Informação (Cetic) (2020), ligado ao Comitê Gestor da Internet do Brasil, 74\% da população brasileira em 2018 estava conectada à internet. Dados divulgados pela OMS (WHO, 2019b) apontam que, em países desenvolvidos, cerca de $88 \%$ das pessoas estavam conectadas. Mais do que entre países, em geral, essas desigualdades são evidentes entre classes sociais. Nas camadas mais pobres da população - categorizadas no estudo em "D" e "E" -, apenas $48 \%$ das pessoas têm acesso à internet, sendo a maior parte (63\%) por smartphones. Portanto, não só acesso, mas também a disponibilidade de consumo de pacote de dados das operadoras (4G) é um obstáculo à disseminação do uso da TS no Brasil. Sem dúvida, a ampliação do acesso a cuidados via TS no SUS exigirá um aporte significativo de recursos em tecnologia de informação e comunicação, o que, nitidamente, ainda não foi feito. Será necessário forte investimento em aquisição de hardwares e softwares e contratação e treinamento de pessoal especializado para suporte e manutenção.

\section{Considerações finais}

A TS é uma valiosa ferramenta para a oferta de cuidados em saúde em situações que exigem o distanciamento social, como 
a enfrentada na pandemia de COVID-19. Desse modo, não há dúvida de que deve fazer parte das ações desenvolvidas no âmbito dos sistemas de saúde como forma de ampliar o acesso. A TS pode facilitar o atendimento oportuno de indivíduos pelos serviços de saúde, apoiar o manejo clínico de pacientes, além de reforçar as medidas de distanciamento social, beneficiando os pacientes e os profissionais de saúde na linha de frente do combate à pandemia.

Além dos benefícios do uso dessa nova tecnologia, é preciso ter em mente os desafios e, sobretudo, os riscos envolvidos que incluem o desrespeito à privacidade, o comprometimento da qualidade da atenção e o aumento das iniquidades em saúde. Por princípio, o uso da TS não deve comprometer a segurança do paciente, a qualidade do atendimento ou a igualdade do acesso. A única exceção aceitável entre o atendimento presencial e o atendimento por meio da TS se refere ao exame físico.

Neste sentido, é fundamental realizar rigorosos estudos avaliativos do desempenho dos serviços oferecidos via TS para que, caso se confirme a expectativa positiva, planejar a expansão do seu uso para em todas as modalidades possíveis.

Considerando o contexto nacional, para produzir benefícios e evitar que os riscos se transformem em danos, apontam-se as seguintes recomendações sobre os usos da TS:

1. Divulgação ampla para a população dos serviços que estão acessíveis através da TS e das suas formas de acesso, especialmente para que seja evitado o uso desnecessário das unidades de emergência para questões não críticas;

2. Garantia, pelos gestores de saúde, da infraestrutura necessária para a conectividade entre profissionais e usuários de serviços de saúde, inclusive por meio de smartphones;

3. Treinamento dos profissionais de saúde para realizar o atendimento à distância como estratégia de atenção; 
4. Observação, pelos profissionais de saúde, de normas institucionais, protocolos e mecanismos de garantia de qualidade, incluindo notificação rápida de eventos adversos, documentação adequada e acompanhamento dos casos de maior gravidade;

5. Garantia da segurança dos dados e das imagens dos pacientes, prezando pela integridade, veracidade, confidencialidade, privacidade e sigilo profissional das informações.

\section{Referências}

ADEPOJU, P. Tuberculosis and HIV responses threatened by COVID-19.

The Lancet HIV, Amsterdam, v. 7, n. 5, p. e319-e320, 2020. DOI: 10.1016/

S2352-3018(20)30109-0. Disponível em: https://www.thelancet.com/journals/

lanhiv/article/PIIS2352-3018(20)30109-0/fulltext Acesso em: 14 abr. 2020.

AHN, D. T. The COVID-19 Pandemic: A "Tech"-tonic Shift Toward

Virtual Diabetes Care. Journal of Diabetes Science and Technology,

London. v. 14, n. 4, p. 708-709, 2020. Disponível em: https://journals.sagepub.com/ doi/10.1177/1932296820929719. Acesso em: 29 maio 2020.

ALLOCCA, M. et al. Maintaining the Quality Standards of Care for Inflammatory Bowel Disease Patients During the COVID-19 Pandemic. Clinical Gastroenterology and Hepatology, Philadelphia, v. 18, n. 8, p. 1882-1883, July 2020. DOI: 10.1016/j. cgh.2020.04.028. Disponível em: https://www.cghjournal.org/article/ S1542-3565(20)30524-3/fulltext Acesso em: 20 maio 2020.

ALSHARAYDEH, I. et al. Challenges and solutions for maternity and gynecology services during the COVID-19 crisis in Jordan. International Journal of Gynecology \& Obstetrics, New York, v. 150, n. 2, p. 159-162, Aug. 2020. DOI: 10.1002/ijgo.13240. Disponível em: https://obgyn.onlinelibrary.wiley.com/doi/full/10.1002/ijgo.13240. Acesso em: 7 jun. 2020. 
ANDERSON, K. et al. Physician's Perceptions of Telemedicine in HIV Care Provision: A Cross-Sectional Web-Based Survey. JMIR Public Health and Surveillance, Toronto, v. 3, n. 2, p. e31, 2017. DOI: 10.2196/publichealth.6896. Disponível em: https://publichealth.jmir.org/2017/2/e31/.

Acesso em: 15 maio 2020.

ANDREWS, J. A. et al. Cuidados e pesquisa em esclerose lateral amiotrófica nos EUA durante a pandemia de COVID-19: desafios e oportunidades. Músculo e Nervo, 2020.

ANDREWS, S. M. et al. Patient perceptions of a comprehensive telemedicine intervention to address persistent poorly controlled diabetes. Patient preference and Adherence, Auckland, v. 11, p. 469-478, 2017. https://doi.org/10.2147/PPA. S125673. Disponível em: https://www.dovepress.com/patient-perceptions-ofa-comprehensive-telemedicine-intervention-to-ad-peer-reviewed-fulltextarticle-PPA. Acesso em: 26 maio 2020.

ARMITAGE, R.; NELLUMS, L. B. Protecting health workers' mental health during COVID-19. Public Health, London, v. 185, p. 18, 2020. Disponível em: https://doi.org/10.1016/j.puhe.2020.05.044. Acesso em: 16 jun. 2020.

ASHFAQ, A. et al. Knowledge and Attitude Regarding Telemedicine Among Doctors in Karachi. Cureus, Palo Alto, v. 12, n. 2, p. e6927, 2020. DOI: 10.7759/cureus.6927. Disponível em: https://doi.org/10.7759/cureus.6927. Acesso em: 30 maio 2020

AMERICAN TELEMEDICINE ASSOCIATION. Telemedicine, Telehealth, and Health Information Technology: An ATA Issue Paper, [s. I.], May 2006. Disponível em: https://www.who.int/goe/policies/countries/usa_support_tele.pdf?ua=1. Acesso em: 16 jun. 2020.

AYATOLLAHI, H.; SARABI, F. Z. P.; LANGARIZADEH, M. Clinicians' knowledge and perception of telemedicine technology. Perspectives In Health Information Management, Chicago, Nov. 2015. Disponível em: https://www.ncbi.nlm.nih.gov/ pmc/articles/PMC4632872/. Acesso em: 30 maio 2020.

AZIZ, A. et al. Telehealth for High-Risk Pregnancies in the Setting of the COVID-19 Pandemic. American Journal of Perinatology, New York, v. 37, n. 8, p. 800-808, June 2020. DOI: 10.1055/s-0040-1712121. Disponível em: https://www. thieme-connect.com/products/ejournals/abstract/10.1055/s-0040-1712121. Acesso em: 17 jun. 2020. 
AZIZY, A; FAYAZ, M.; AGIRBASLI, M. Do Not Forget Afghanistan in Times of COVID-19: Telemedicine and the Internet of Things to Strengthen Planetary Health Systems. OMICS: A Journal of Integrative Biology Ahead of Print, Larchmont, NY, v. 24, n. 6, p. 311-31, 2020. DOI: https://doi.org/10.1089/omi.2020.0053. Disponível em: https://doi.org/10.1089/omi.2020.0053. Acesso em: 15 jun. 2020.

BADAWY, S. M.; RADOVIC, A. Digital Approaches to Remote Pediatric Health Care Delivery During the COVID-19 Pandemic: Existing Evidence and a Call for Further Research. JMIR Pediatrics and Parenting, Toronto, v. 3, n. 1, p. e20049, 2020. DOI: https://doi.org/10.2196/20049. Disponível em: https://pediatrics.jmir. org/2020/1/e20049/. Acesso em: 7 jul. 2020.

BAHIA. Secretaria da Saúde. Tele Coronavírus 155 começa a funcionar para atender a população gratuitamente na Bahia. Salvador, 24 mar. 2020. Disponível em: http:// www.saude.ba.gov.br/2020/03/24/tele-coronavirus-155-comeca-a-funcionarpara-atender-a-populacao-gratuitamente-na-bahia/. Acesso em: 10 maio 2020.

BARNEY, A. et al. The COVID-19 Pandemic and Rapid Implementation of Adolescent and Young Adult Telemedicine: Challenges and Opportunities for Innovation. The Journal Of Adolescent Health: Official Publication Of The Society For Adolescent Medicine, New York, v. 67, n. 2, p. 164-171, 2020. DOI: https://doi. org/10.1016/j.jadohealth.2020.05.006. Disponível em: https://www.jahonline.org/ article/S1054-139X(20)30225-1/fulltext. Acesso em: 7 jun. 2020.

BARTELS, S. J. et al. COVID-19 Emergency Reforms in Massachusetts to Support Behavioral Health Care and Reduce Mortality of People With Serious Mental Illness. Psychiatric Services, v. 71, n. 10, p. 1078-1081. 2020. Disponível em: https:// ps.psychiatryonline.org/doi/10.1176/appi.ps.202000244. Acesso em: 17 jun. 2020.

LIN, B.; WU, S. J. COVID-19 (Coronavirus Disease 2019): Opportunities and Challenges for Digital Health and the Internet of Medical Things in China. OMICS: A Journal of Integrative Biology, Larchmont, NY, v. 24, n. 5, p. 231-232, May 2020. DOI: http://doi.org/10.1089/omi.2020.0047. Disponível em: https://www. liebertpub.com/doi/abs/10.1089/omi.2020.0047. Acesso em: 22 maio 2020.

BRADFORD, N. K.; CAFFERY, L. J.; SMITH, A. C. Telehealth services in rural and remote Australia: a systematic review of models of care and factors influencing success and sustainability. Rural Remote Health, Townsville, v. 16, n. 4, p. 3808, Oct./Dec. 2016. Disponível em: https://www.rrh.org.au/articles/ subviewnew.asp?ArticlelD=3808. Acesso em: 15 maio 2020 
BRASIL. Casa Civil. TeleSUS fará busca ativa de informações sobre coronavírus. Brasília, DF, 1 abr. 2020a. Disponível em: https://www.saude.gov.br/noticias/ agencia-saude/46633-ministerio-da-saude-fara-busca-ativa-de-informacoessobre-coronavirus. Acesso em: 24 abr. 2020.

BRASIL. Ministério da Saúde. Gabinete do Ministro. Portaria n 2.546, de 27 de outubro de 2011. Redefine e amplia o Programa Telessaúde Brasil, que passa a ser denominado Programa Nacional Telessaúde Brasil Redes (Telessaúde Brasil Redes). Diário Oficial da União: seção 1, Brasília, DF, p. 50-51, 28 out. 2011. Disponível em: https://bvsms.saude.gov.br/bvs/saudelegis/gm/2011/prt2546_27_10_2011.html. Acesso em: 24 abr. 2020.

BRASIL. Ministério da Saúde. Portaria n 467, de 20 de março de 2020. Dispõe, em caráter excepcional e temporário, sobre as ações de Telemedicina, com o objetivo de regulamentar e operacionalizar as medidas de enfrentamento da emergência de saúde pública de importância internacional previstas no art. $3^{\circ}$ da Lei $\mathrm{n}^{\circ} 13.979$, de 6 de fevereiro de 2020, decorrente da epidemia de COVID19. Diário Oficial da União: seção 1, Brasília, DF, n. 56-B, p. 1, 2 mar. 2020b. Disponível em: https://www.in.gov.br/en/web/dou/-/portaria-n-467-de-20-demarco-de-2020-249312996. Acesso em: 24 abr. 2020.

BRASIL. Ministério da Saúde. Comitê Gestor da Estratégia e-Saúde. Estratégia e-saúde para o Brasil. Brasília, DF, 2017a. Disponível em: https://saudedigital. saude.gov.br/wp-content/uploads/2020/02/Estrategia-e-saude-para-o-Brasil_ CIT_20170604.pdf. Acesso em: 5 maio 2020.

BRASIL. Ministério da Saúde. Resolução n 19, de 22 de junho de 2017. Aprova e torna público o documento Estratégiae-Saúde para o Brasil. Diário Oficial da União: seção 1, Brasília, DF, n. 113, p. 130, 13 jul. 2017b. Disponível em: https:// www.in.gov.br/materia/-/asset_publisher/Kujrw0TZC2Mb/content/id/19172500/ do1-2017-07-13-resolucao-n-19-de-22-de-junho-de-2017-19172419.

Acesso em: 5 maio 2020.

BRASIL. Ministério da Saúde. Resolução n 46, de 29 de agosto de 2019. Institui o Comitê Gestor da Estratégia de Saúde Digital e define a sua composição, as suas competências e as suas unidades operacionais na estrutura do Ministério da Saúde, em substituição ao Comitê Gestor da Estratégia de e-Saúde no Brasil. Diário Oficial da União: seção 1, Brasília, DF, n. 198, p. 49, 11 out. 2019a. Disponível em: https:// www.in.gov.br/web/dou/-/resolucao-n-46-de-29-de-agosto-de-2019-221309239. Acesso em: 23 maio 2020. 
BRASIL. Ministério da Saúde. Resolução n 46, de 29 de agosto de 2019. Institui o Comitê Gestor da Estratégia de Saúde Digital e define a sua composição, as suas competências e as suas unidades operacionais na estrutura do Ministério da Saúde, em substituição ao Comitê Gestor da Estratégia de e-Saúde no Brasil. Diário Oficial da União: seção 1, Brasília, DF, n. 198, p. 49,v. 30, n. 7, p. 2043, 2019 b. Disponível em: https://www.in.gov.br/web/dou/-/resolucao-n-46-de-29-deagosto-de-2019-221309239. Acesso em: 23 maio 2020.

BRYANT, M. S.; FEDSON, S. E.; SHARAFKHANEH, A. Using Telehealth Cardiopulmonary Rehabilitation during the COVID-19 Pandemic. Journal of Medical Systems, New York, v. 44, n. 7, p. 125, 2020. DOI: 10.1007/ s10916-020-01593-8. Disponível em: https://link.springer.com/article/10.1007/ s10916-020-01593-8. Acesso em: 10 jun. 2020.

BUCKSTEIN, M. et al. Experiencing the Surge: Report from a Large New York Radiation Oncology Department During the COVID-19 Pandemic. Advances in Radiation Oncology, Philadelphia, v. 5, n. 4, p. 610-616. 2020. DOI:10.1016/j. adro.2020.04.014. Disponível em: https://www.advancesradonc.org/article/ S2452-1094(20)30090-7/fulltext Acesso em: 20 maio 2020.

CAETANO, R. et al. Challenges and opportunities for telehealth during the COVID-19 pandemic: ideas on spaces and initiatives in the Brazilian context. Caderno de Saúde Pública, Rio de Janeiro, v. 36, n. 5, 2020. DOI: https://doi. org/10.1590/0102-311x00088920. Disponível em http://www.scielo.br/scielo. php?script=sci_arttext\&pid=S0102-311X2020000503001\&lng=en\&nrm=iso. Acesso em: 16 jun. 2020.

CAFFERY, L. J. Telehealth interventions for reducing waiting lists and waiting times for specialist outpatient services: a scoping review. Journal of Telemedicine and Telecare, London, v. 22, n. 8, p. 504-512, 2016. DOI: 10.1177/1357633X16670495. Disponível em: https://journals.sagepub.com/doi/10.1177/1357633X16670495? url_ver=Z39.88-2003\&rfr_id=ori:rid:crossref.org\&rfr_dat=cr_pub\%20\%200pubmed Acesso em: 6 jun. 2020.

CALTON, B.; ABEDINI, N.; FRATKIN, M. Telemedicine in the Time of Coronavirus. Journal of Pain and Symptom Management, Madison, v. 60, n. 1, p. e12-e14, 2020. DOI: https://doi.org/10.1016/j.jpainsymman.2020.03.019. Disponível em: https:// linkinghub.elsevier.com/retrieve/pii/S0885392420301706. Acesso em: 17 jul. 2020.

CENTRO REGIONAL DE ESTUDOS PARA O DESENVOLVIMENTO DA SOCIEDADE DA INFORMAÇÃO - CETIC. TIC Domicílios. Disponível em http://data.cetic.br/cetic/ dados?idPesquisa=TIC_DOM\&idUnidadeAnalise=Usuarios. Acesso em: 4 jun. 2020. 
CHAUHAN, V. et al. Novel Coronavirus (COVID-19): Leveraging Telemedicine to Optimize Care While Minimizing Exposures and Viral Transmission. Journal of Emergencies, Trauma, And Shock, Mumbai, v. 13, n. 1, p. 20-24, 2020. DOI:10.4103/ JETS.JETS_32_20. Disponível em: https://www.ncbi.nlm.nih.gov/pmc/articles/ PMC7161346/. Acesso em: 5 maio 2020.

CHEN, Q. et al. Mental health care for medical staff in China during the COVID-19 outbreak. Lancet Psychiatry, Kidlington, v. 7, n. 4, p. e15-e16, Apr. 2020. DOI: 10.1016/S2215-0366(20)30078-X. Disponível em: https://www.thelancet.com/ journals/lanpsy/article/PIIS2215-0366(20)30078-X/fulltext Acesso em: 3 jun. 2020.

CHENG, P. et al. COVID-19 Epidemic Peer Support and Crisis Intervention Via Social Media. Community Mental Health Jornal, New York, v. 56, n. 6, p. 786-792, 2020. DOI: https://doi.org/10.1007/s10597-020-00624-5. Disponível em: https:// link.springer.com/article/10.1007/s10597-020-00624-5. Acesso em: 20 maio 2020.

CHOWDHURY, D. et al. Telehealth for Pediatric Cardiology Practitioners in the Time of COVID-19. Pediatric Cardiology, New York, v. 41, n. 6, p. 1081-1091, 2020. Advance online publication. DOI: https://doi.org/10.1007/s00246-020-02411-1. Disponível em: https://link.springer.com/article/10.1007/s00246-020-02411-1. Acesso em: 4 jul. 2020.

CONSELHO FEDERAL DE MEDICINA (Brasil) - CFM. Resolução CFM n 1.643/2002. Define e disciplina a prestação de serviços através da Telemedicina. Diário Oficial da União: seção 1, Brasília, DF, p. 205, 26 ago. 2019a.

CONSELHO FEDERAL DE MEDICINA (Brasil) - CFM. Resolução CFM n 2.227/2018. Define e disciplina a telemedicina como forma de prestação de serviços médicos mediados por tecnologias. Diário Oficial da União: seção 1, Brasília, DF, p. 58, 6 fev. 2019b. Disponível em: https://portal.cfm.org.br/images/PDF/ resolucao222718.pdf Acesso em: 13 jun. 2020.

CONSELHO FEDERAL DE MEDICINA (Brasil) - CFM. Ofício CFM n 1756/2020 - CONJUR. Brasília, DF, 19 mar. 2020. Disponível em: http://portal.cfm.org.br/ images/PDF/2020_oficio_telemedicina.pdf. Acesso em: 14 abr. 2020.

DIGIOVANNI, G. et al. Development of a telehealth geriatric assessment model in response to the COVID-19 pandemic. Journal of Geriatric Oncology, Amsterdam, v. 11, n. 5, p. 761-763, 2020. https://doi.org/10.1016/j.jgo.2020.04.007.

Disponível em: https://www.geriatriconcology.net/article/S1879-4068(20)30200-9/ fulltext. Acesso em: 15 jun. 2020. 
DING, X. et al. Wearable Sensing and Telehealth Technology with Potential Applications in the Coronavirus Pandemic. IEEE Reviews in Biomedical Engineering, Piscataway, NJ, 2020. DOI: 10.1109/RBME.2020.2992838. Disponível em: https:// ieeexplore.ieee.org/document/9090987 Acesso em: 3 jun. 2020.

DOURADO, I. et al. Adapting to the COVID-19 Pandemic: Continuing HIV Prevention Services for Adolescents Through Telemonitoring, Brazil. AIDS and Behavior, New York, v. 24, n. 7, p. 1994-1999, 2020.

DU TOIT, M. et al. Use of telehealth in the management of non-critical emergencies in rural or remote emergency departments: a systematic review. Journal of Telemedicine and Telecare, London, v. 25, n. 1,

p. 3-16, 2019. DOI: 10.1177/1357633X17734239. Disponível em: https://doi. org/10.1177/1357633X17734239. Acesso em: 6 maio 2020.

EDELMAN, L. S. et al. Mitigating the Effects of a Pandemic: Facilitating Improved Nursing Home Care Delivery Through Technology. JMIR Aging, Toronto, v. 3, n. 1, p. e20110, May 2020. DOI: 10.2196/20110. Disponível em: https://aging.jmir. org/2020/1/e20110/. Acesso em: 13 jul. 2020.

ELKADDOUM, R. et al. Telemedicine for cancer patients during COVID-19 pandemic: between threats and opportunities. Future Oncology, London, v. 16, n. 18, p. 1225-1227, 2020. DOI: https://doi.org/10.2217/fon-2020-0324. Disponível em: https://www.futuremedicine.com/doi/10.2217/fon-2020-0324. Acesso em: 16 jul. 2020.

FOSARELLI, P. D. The Telephone in Pediatric Medicine. Clinical Pediatrics, Thousand Oaks, v. 22, n. 4, p. 293-296, 1983. Disponível em: http://journals. sagepub.com/doi/10.1177/000992288302200409. Acesso em: 13 jul. 2020.

FUKUTI, P. et al. How Institutions Can Protect the Mental Health and Psychosocial Well-Being of Their Healthcare Workers in the Current COVID-19 Pandemic. Clinics, São Paulo, v. 75, 2020. Disponível em: https://doi.org/10.6061/clinics/2020/ e1963. Acesso em: 20 jun. 2020.

FUNDACENTRO. Até 10\% dos profissionais da saúde são atingidos por COVID19. 2020. Disponível em: http://www.fundacentro.gov.br/noticias/detalhe-danoticia/2020/3/ate-10-dos-profissionais-da-saude-sao-atingidos-por-covid-19. Acesso: 4 jun. 2020. 
GHOSH, A.; GUPTA, R.; MISRA, A. Telemedicine for diabetes care in India during COVID19 pandemic and national lockdown period: Guidelines for physicians. Diabetes \& Metabolic Syndrome, Amsterdam, v. 14, n. 4, p. 273-276, 2020. DOI: 10.1016/j.dsx.2020.04.001. Disponível em: https://doi.org/10.1016/j. dsx.2020.04.001. Acesso em: 20 maio 2020.

GOODMAN-CASANOVA, J. M. et al. Telehealth Home Support During COVID-19 Confinement for Community-Dwelling Older Adults With Mild Cognitive Impairment or Mild Dementia: Survey Study. Journal of Medical Internet Research, Pittsburgh, v. 22, n. 5, p. e19434, 2020. DOI: 10.2196/19434. Disponível em: https://doi.org/10.1016/j.dsx.2020.04.001. Acesso em: 4 jun. 2020,

GONG, K. et al. Internet Hospitals Help Prevent and Control the Epidemic of COVID-19 in China: Multicenter User Profiling Study. Journal of Medical Internet Research, Pittsburgh, v. 22, n. 4, p. e18908. DOI: 10.2196/18908. Disponível em: https://www.jmir.org/2020/4/e18908/. Acesso em: 10 maio 2020.

GREENHALGH, T. et al. Video consultations for covid-19. BMJ, London, n. 368, p. m998, 2020. Disponível em: https://www.bmj.com/content/368/bmj.m998. Acesso em: 23 abr. 2020.

HIRKO, K. A. et al. Telehealth in Response to the Covid-19 Pandemic: Implications for Rural Health Disparities. Journal of the American Medical Informatics Association, Philadelphia, 2020. DOI:1 0.1093/jamia/ocaa156. Disponível em: https://academic.oup.com/jamia/article/27/11/1816/5863253. Acesso em: 6 jul. 2020.

HOAGLAND, B. et al. Telemedicine as a tool for PrEP delivery during the COVID-19 Pandemic in a large HIV Prevention service in Rio de Janeiro-Brazil. The Brazilian Journal of Infectious Diseases, Salvador, v. 24, n. 4, p. 360-364, 2020. Disponível em: https://www.bjid.org.br/en-telemedicine-as-tool-for-preparticulo-S1413867020300659. Acesso em: 6 jul. 2020.

HOLLANDER, J. E.; CARR, B. G. Virtually Perfect? Telemedicine for Covid-19. The New England Journal of Medicine, Boston, v. 382, n. 18, p. 1679-1681, 2020. DOI: 10.1056/NEJMp2003539. Disponível em: https://www.nejm.org/doi/ full/10.1056/nejmp2003539. Acesso em: 4 jun. 2020.

HONG, Z. et al. Telemedicine During the COVID-19 Pandemic: Experiences From Western China. Journal of Medical Internet Research, Pittsburgh, v. 22, n. 5, p. e19577, 2020. DOI: 10.2196/19577. Disponível em: https://www.jmir. org/2020/5/e19577/. Acesso em: 20 maio 2020. 
HUMPHREYS, J. et al. Rapid Implementation of Inpatient Telepalliative Medicine Consultations During COVID-19 Pandemic. Journal of Pain and Symptom Management, Madison, v. 60, n. 1, p. e54-e59, 2020. DOI: 10.1016/j. jpainsymman.2020.04.001. Disponível em: https://www.jpsmjournal.com/article/ S0885-3924(20)30195-0/fulltext. Acesso em: 17 jul. 2020.

JOHN, O. Video consultations for triage of patients with covid-19. BMJ, London, n. 369, p. m1583, 2020. DOI: https://doi.org/10.1136/bmj. m1583. Disponível em: https://www.bmj.com/content/369/bmj.m1583. Acesso em: 10 maio 2020.

KANG, L. et al. The mental health of medical workers in Wuhan, China dealing with the 2019 novel coronavirus. Lancet Psychiatry, Kidlington, v. 7, n. 3, p. e14, 2020. DOI: 10.1016/S2215-0366(20)30047-X. Disponível em: https://www. thelancet.com/journals/lanpsy/article/PIIS2215-0366(20)30047-X/fulltext. Acesso em: 27 abr. 2020.

KANNEGANTI, A. et al. Continuing medical education during a pandemic: an academic institution's experience. Postgraduate Medical Journal, London, v. 96, n. 1137, p. 384-386, 2020.

KARPOV, K. Ministério da Saúde garante suporte psicológico a profissionais do SUS. Política Distrital, [Brasília, DF], 22 abr. 2020. Disponível em: https://www.saude. gov.br/noticias/agencia-saude/46767-ministerio-da-saude-garante-suportepsicologico-a-profissionais-do-sus. Acesso em: 4 jun. 2020.

KESHVARDOOST, S.; BAHAADINBEIGY, K.; FATEHI, F. Role of Telehealth in the Management of COVID-19: Lessons Learned from Previous SARS, MERS, and Ebola Outbreaks. Telemedicine Journal and E-Health, Larchmont, v. 26, n. 7, p. 850-852, 2020. DOI: 10.1089/tmj.2020.0105. Disponível em: https://www.liebertpub.com/ doi/full/10.1089/tmj.2020.0105. Acesso em: 17 jul. 2020.

KHATRI, U. G.; PERRONE, J. Opioid Use Disorder and COVID-19: Crashing of the Crises. Journal of Addiction Medicine, Hagerstown, v. 14, n. 4, p. e6-e7, 2020. DOI: 10.1097/ADM.0000000000000684. Disponível em: https://www.ncbi.nlm.nih. gov/pmc/articles/PMC7236857/. Acesso em: 5 jun. 2020.

KIBERU, V. M.; SCOTT, R. E.; MARS, M. Assessing core, e-learning, clinical and technology readiness to integrate telemedicine at public health facilities in Uganda: a health facility - based survey. BMC health Services Research, London, v. 19, n. 1, p. 266, 2019. DOI: 10.1186/s12913-019-4057-6. Disponível em: https://bmchealthservres.biomedcentral.com/articles/10.1186/s12913-019-4057-6. Acesso em: 10 maio 2020. 
KRAUSZ, M. et al. Emergency Response to COVID-19 in Canada: Platform Development and Implementation for eHealth in Crisis Management. JMIR Public Health and Surveillance, Toronto, v. 6, n. 2, 2020. Disponível em: https://www.ncbi. nlm.nih.gov/pmc/articles/PMC7236607/. Acesso em: 30 maio 2020.

KUMMITHA, R. K. R. Smart technologies for fighting pandemics: The techno- and human- driven approaches in controlling the virus transmission. Goverment Information Quarterly, Greenwich, v. 3, n. 3, p. 101481, 2020. DOI: 10.1016 / j.giq.2020.101481 Disponível em: https://www.sciencedirect. com/science/article/pii/S0740624X20301003. Acesso em: 26 jun. 2020.

LADITKA, J. N.; LADITKA, S. B.; PROBST, J. C. Health care access in rural areas: evidence that hospitalization for ambulatory care-sensitive conditions in the United States may increase with the level of rurality. Health \& Place, Oxford, v. 15, p. 731-740, 2009. DOI: 10.1016/j.healthplace.2008.12.007 3. Disponível em: https:// www.sciencedirect.com/science/article/abs/pii/S1353829209000057?via\%3Dihub. Acesso em: 30 maio 2020.

LAKKIREDDY, D. R. et al. Guidance for Cardiac Electrophysiology During the COVID-19 Pandemic from the Heart Rhythm Society COVID-19 Task Force; Electrophysiology Section of the American College of Cardiology; and the Electrocardiography and Arrhythmias Committee of the Council on Clinical Cardiology, American Heart Association. Circulation, Dallas, v. 141, n. 21, p. e823-e831, 2020.

LAKKIREDDY, D. R. et al. Guidance for cardiac electrophysiology during the COVID-19 pandemic from the Heart Rhythm Society COVID-19 Task Force; Electrophysiology Section of the American College of Cardiology; and the Electrocardiography and Arrhythmias Committee of the Council on Clinical Cardiology, American Heart Association. Heart Rhythm, New York, v. 17, n. 9, p. e233-e241, 2020. Disponível em: https://doi.org/10.1016/j. hrthm.2020.03.028. Disponível em: https://www.heartrhythmjournal.com/article/ S1547-5271(20)30289-7/fulltext. Acesso em: 5 maio 2020

LATTIMER, V. et al. Safety and effectiveness of nurse telephone consultation in out of hours primary care: randomised controlled trial. The South Wiltshire Out of Hours Project (SWOOP) Group. BMJ, London, v. 317, n. 7165, p. 1054-1059, 1998. DOI: 10.1136/bmj.317.7165.1054. Disponível em: https://www.bmj.com/ content/317/7165/1054. Acesso em: 16 abr. 2020. 
LEE, I. et al. Lipoff Telehealth: Helping Your Patients and Practice Survive and Thrive During the COVID-19 Crisis with Rapid Quality Implementation. Journal of the American Academy of Dermatology, St. Louis, v. 82, n. 5, p. 1213-1214 2020.

LEES, C. W.; REGUEIRO, M.; MAHADEVAN, U. Innovation in IBD Care During the COVID-19 Pandemic: Results of a Global Telemedicine Survey by the International Organization for the Study of Inflammatory Bowel Disease. Gastroenterology, Philadelphia, v. 159, n. 3, p. 805-808, 2020. DOI: 10.1053/j. gastro.2020.05.063. Disponível em: https://doi.org/10.1053/j.gastro.2020.05.063. Acesso em: 20 jun. 2020.

LEWIS, G. D. et al. Long-term Institutional Experience with Telemedicine Services for Radiation Oncology: A Potential Model for Long-term Utilization. Advances in Radiation Oncology, Philadelphia, v. 5, n. 4, p. 780-782, 2020. DOI: 10.1016/j. adro.2020.04.018. Disponível em: https://www.advancesradonc.org/article/ S2452-1094(20)30094-4/fulltext Acesso em: 30 maio 2020.

LOH, T. Y.; HSIAO, J. L.; SHI, V. Y. COVID-19 and its effect on medical student education in dermatology. Journal of the American Academy of Dermatology, St. Louis, v. 83, n. 2, p. e163-e164, 2020. Disponível em: https://www. jaad.org/article/S0190-9622(20)30857-4/fulltext. Acesso em: 30 maio 2020.

LOOI, J. C. L; PRING, W. Private metropolitan telepsychiatry in Australia during Covid-19: current practice and future developments. Australasian Psychiatry, Carlton, v. 28, n. 5, p. 508-510, 2020. DOI: 10.1177/1039856220930675. Disponível em: https://doi.org/10.1177/1039856220930675 Acesso em: 26 jun. 2020.

MANN, D. M. et al. COVID-19 transforms health care through telemedicine: evidence from the field. Journal of the American Medical Informatics Association, Philadelphia, v. 27, n. 7, p. 1132-1135, 2020.

MASSEY, P. A. et al. Orthopaedic Surgical Selection and Inpatient Paradigms During the Coronavirus (COVID-19) Pandemic. The Journal of the American Academy of Orthopaedic Surgeons, Rosemont, v. 28, n. 11, p. 436-450, 2020. DOI: 10.5435/JAAOS-D-20-00360. Disponível em: https://journals.Iww.com/ jaaos/Fulltext/2020/06010/Orthopaedic_Surgical_Selection_and_Inpatient.4.aspx. Acesso em: 5 maio 2020. 
MELONI, M. et al. Management of diabetic persons with foot ulceration during COVID-19 health care emergency: Effectiveness of a new triage pathway. Diabetes Research and Clinical Practice, Amsterdam, 2020. DOI: https:// doi.org/10.1016/j.diabres.2020.108245. Disponível em: https://www. diabetesresearchclinicalpractice.com/article/S0168-8227(20)30495-2/fulltext. Acesso em: 17 jun. 2020.

MENON, V.; PADHY, S. K. Ethical dilemmas faced by health care workers during COVID-19 pandemic: Issues, implications and suggestions. Asian journal of psychiatry, Amsterdam, v. 51, p. 102116, 2020. Advance online publication. https:// doi.org/10.1016/j.ajp.2020.102116. Disponível em: https://www.sciencedirect.com/ science/article/abs/pii/S1876201820302276?via\%3Dihub. Acesso em: 5 maio 2020.

MGBAKO, O. et al. COVID-19, Telemedicine, and Patient Empowerment in HIV Care and Research. AIDS and Behavior, New York, v. 24, n. 7, p. 1990-1993, 2020.

NEUBECK, L. et al. Delivering healthcare remotely to cardiovascular patients during COVID-19: A rapid review of the evidence. European Journal of Cardiovascular Nursing, Amsterdam, v. 19, n. 6, p. 486-494, 2020. DOI: 10.1177/1474515120924530. Disponível em: https://journals.sagepub.com/ doi/full/10.1177/1474515120924530. Acesso em: 30 maio 2020.

NGUYEN, N. P. et al. Older Cancer Patients during the COVID-19 Epidemic: Practice Proposal of the International Geriatric Radiotherapy Group. Cancers, Basel, v. 12, n. 5, p. 1287, 2020. DOI: https://doi.org/10.3390/cancers12051287. Acesso em: 26 jun. 2020.

NORRIS, A. C. Essentials of Telemedicine and Telecare. Baffins Lane: John Wiley \& Sons, 2002. DOI: 10.1002/0470846348. Disponível em: https://onlinelibrary.wiley. com/doi/pdf/10.1002/0470846348.fmatter_indsub. Acesso em: 30 maio 2020.

ODA, D. S.; HEILBRON, D. C.; TAYLOR, H. J. A preventive child health program: The effect of telephone and home visits by public health nurses. American Journal of Public Health, New York, v. 85, n. 6, p. 854-855, 1995.

OMBONI, S. Telemedicine During The COVID-19 in Italy: A Missed Opportunity? Telemedicine Journal and E-Health, Larchmont, v. 26, n. 8, p. 973-975, 2020. DOI: https://doi.org/10.1089/tmj.2020.0106. Disponível em: https://www. liebertpub.com/doi/full/10.1089/TMJ.2020.0106. Acesso em: 6 ago. 2020.

ORGANIZAÇÃO PAN-AMERICANA DA SAÚDE - OPAS. Campus Virtual de Saúde Pública da OPAS/OMS. 2020a. Disponível em: https://www.campusvirtualsp.org/ pt-br. Acesso em: 17 maio 2020 
ORGANIZAÇÃO PAN-AMERICANA DA SAÚDE - OPAS. Folha informativa COVID-19 - Escritório da OPAS e da OMS no Brasil. 30 out. 2020b.

Disponível em: https://www.paho.org/bra/index.php?option=com_ content\&view=article\&id=6101: covid19\&Itemid=875. Acesso em: 17 maio 2020.

O'HARA, V. M.; JOHNSTON, S. V.; BROWNE, N. T. The paediatric weight management office visit via telemedicine: pre- to post-COVID-19 pandemic.

Pediatric Obesity, Oxford, v. 15, n. 8, p. e12694, 2020. DOI: https://doi.org/10.1111/ ijpo.12694. Disponível em: https://europepmc.org/article/med/32627434. Acesso em: 23 maio 2020.

PALADINO, L. et al. Reflections on the ebola public health emergency of international concern, part 2: The unseen epidemic of posttraumatic stress among health-care personnel and survivors of the 2014-2016 Ebola outbreak. Journal of Global Infectious Diseases, Mumbai, v. 9, n. 2, p. 45-50, Apr./ June 2017. Disponível em: http://www.jgid.org/text.asp?2017/9/2/45/206945. Acesso em: 5 jun. 2020.

PAPADIMOS, T. J. et al. Ethics of Outbreaks Position Statement. Part 1: Therapies, Treatment Limitations, and Duty to Treat. Critical Care Medicine, Philadelphia, v. 46, n. 11, p. 1856-1860, Nov. 2018. DOI: 10.1097/ccm.0000000000003416. Disponível em: https://journals.lww.com/ccmjournal/Abstract/2018/11000/Ethics_ of_Outbreaks_Position_Statement_Part_1_.16.aspx. Acesso em: 16 maio 2020.

PAPADOPOULOS, N. G. et al. Pediatric Asthma in Real Life Collaborators Impact of COVID-19 on Pediatric Asthma: Practice Adjustments and Disease Burden. The Journal of Allergy And Clinical Immunology. In practice, 2020. DOI: https://doi. org/10.1016/j.jaip.2020.06.001. Disponível em: https://www.sciencedirect.com/ science/article/pii/S2213219820305997?via\%3Dihub. Acesso em: 26 jun. 2020.

PASKUDZKA, D. et al. Telephone follow-up of patients with cardiovascular implantable electronic devices during the coronavirus disease 2019 pandemic: early results. Kardiologia Polska, Warszawa, v. 78, n. 7/8, p. 725-731, 2020. DOI: 10.33963/kp.15392. Disponível em: https://www.mp.pl/kardiologiapolska/ issue/article/15392. Acesso em: 4 jun. 2020.

PEAHL, A. F.; SMITH, R. D.; MONIZ, M. H. Prenatal care redesign: creating flexible maternity care models through virtual care. American Journal of Obstetrics and Gynecology, St. Louis, v. 223, n. 3, p. 389.e1-389.e10, 2020. Disponível em: https://doi.org/10.1016/j.ajog.2020.05.029. Acesso em: 10 jun. 2020. 
PEREZ-ALBA, E. et al. Use of self-administered surveys through QR code and same center telemedicine in a walk-in clinic in the era of COVID-19. Journal of the American Medical Informatics Association, Philadelphia,, v. 27, n. 6, p. 985-986, 2020. DOI: https://doi.org/10.1093/jamia/ocaa054. Disponível em: https://academic.oup. com/jamia/article/27/6/985/5819556. Acesso em: 30 maio 2020.

PEREZ-SUST, P. et al. Turning the crisis into an opportunity: digital health strategies deployed at-scale during the coronavirus (COVID-19) outbreak in Catalonia. JMIR Public Health Surveillance, Toronto, v. 6, n. 2, p. e19106, 2020. DOI: 10.2196/19106. Disponível em: https://publichealth.jmir.org/2020/2/e19106/. Acesso em: 26 maio 2020.

PLOCIENNICZAK, M. J. et al. Guidelines for Resident Participation in Otolaryngology Telehealth Clinics During the COVID-19 Pandemic. OtolaryngologyHead and Neck Surgery, Rochester, v. 163, n. 3, p. 498-500, 2020. Sept. 2020. DOI: https://doi.org/10.1177/0194599820932133. Disponível em: https://journals. sagepub.com/doi/full/10.1177/0194599820932133. Acesso em: 3 jul. 2020.

PLOŃSKA-GOŚCINIAK, E. et al. Management of valvular and structural heart diseases during the coronavirus disease 2019 pandemic: an expert opinion of the Working Group on Valvular Heart Diseases, the Working Group on Cardiac Surgery, and the Association of Cardiovascular Interventions of the Polish Cardiac Society. Kardiologia Polska, Warszawa, v. 78, n. 5, p. 498-507, 2020. DOI: 10.33963/ KP.15358. Disponível em: https://www.mp.pl/kardiologiapolska/issue/article/15358. Acesso em: 4 jun. 2020.

PLUYMAEKERS, N. et al. On-demand app-based rate and rhythm monitoring to manage atrial fibrillation through teleconsultations during COVID-19. International Journal of Cardiology. Heart \& Vasculature, Shannon, 2020. DOI: https://doi. org/10.1016/j.jjcha.2020.100533. Disponível em: https://www.ncbi.nlm.nih.gov/ pmc/articles/PMC7205626/ Acesso em: 15 maio 2020.

PORTNOY, J; WALLER, M.; ELLIOTT, T. Telemedicine in the Era of COVID-19. The Journal Of Allergy And Clinical Immunology. In Practice, New York, v. 8, n. 5, p. 1489-1491, May 2020. DOI: 10.1016/j.jaip.2020.03.008. Disponível em: https:// www.sciencedirect.com/science/article/pii/S221321982030249X?via\%3Dihub. Acesso em: 10 abr. 2020.

POWER, J. D. Telehealth: Best Consumer Healthcare Experience You've Never Tried, Says J.D. Power Study. 2019. Disponível em: https://www.businesswire.com/news/ home/20191028005168/en/Telehealth-Best-Consumer-Healthcare-ExperienceYou\%E2\%80\%99ve-Never-Tried-Says-J.D.-Power-Study. Acesso em: 24 mar. 2020. 
PROBST, J. C. et al. Person and place: the compounding effects of race/ethnicity and rurality on health. American Journal of Public Health, Washington, v. 94, p. 1695-703, 2004. DOI: 10.2105/ajph.94.10.1695. Disponivel em: https://ajph. aphapublications.org/doi/10.2105/AJPH.94.10.1695. Acesso em: 23 maio 2020.

RAHMAN, M. S. et al. Defending against the Novel Coronavirus (COVID-19) Outbreak: How Can the Internet of Things (IoT) help to save the World? Health Policy Technology, Amsterdam, v. 9, n. 2, p. 136-138, 2020. DOI: 10.1016 / j.hlpt.2020.04.005. Disponível em: https://www.sciencedirect.com/science/article/ abs/pii/S221188372030040X?via\%3Dihub. Acesso em: 5 maio 2020.

REEVES, J. J. et al. Rapid Response to COVID-19: Health Informatics Support for Outbreak Management in an Academic Health System. Journal of the American Medical Informatics Association, Philadelphia, v. 27, n. 6, p. 853-859, 2020. Disponível em: https://doi.org/10.1093/jamia/ocaa037 Acesso em: 7 maio 2020 RIPP, J.; PECCORALO, L.; CHARNEY, D. Attending to the Emotional Well-Being of the Health Care Workforce in a New York City Health System During the COVID-19 Pandemic. Academic medicine. Journal of the Association of American Medical Colleges, Philadelphia, v. 95, n. 8, p. 1136-1139, 2020. Disponível em: https://doi. org/10.1097/ACM.0000000000003414. Acesso em: 11 jul. 2020.

ROMANICK-SCHMIEDL, S.; RAGHU, G. Telemedicine - maintaining quality during times of transition. Nature Reviews Disease Primers, [S. I.], v. 6, n. 1, 2020. DOI: 10.1038/s41572-020-0185-x. Disponível em: https://www.nature.com/ articles/s41572-020-0185-x. Acesso em: 13 jun. 2020.

SHAH M.N. et al. A qualitative evaluation of a telemedicine-enhanced emergency care program for older adults. Journal of the American Geriatrics Society, Malden, v. 61, n. 4, p. 571-576, 2013. DOI: 10.1111/jgs.12157. Disponível em: https://www.urmc.rochester.edu/MediaLibraries/URMCMedia/emergencymedicine/research/documents/telemedicine-enhanced-emergency-care.pdf. Acesso em: 17 jul. 2020.

SINGH, G.K.; SIAHPUSH, M. Widening rural-urban disparities in life expectancy, U.S., 1969-2009. American Journal of Preventive Medicine, New York, v. 46, n. 2, p. e19-29, Feb. 2014. DOI: 10.1016/j.amepre.2013.10.017. Disponível em: https://www.ajpmonline.org/article/S0749-3797(13)00590-4/ fulltext. Acesso em: 17 jul. 2020.

SMITH, A.C.; THOMAS, E.; SNOSWELL, C.L., et al. Telehealth for global emergencies: Implications for coronavirus disease 2019 (COVID-19). Journal of Telemedicine and Telecare, London, v. 26, n. 5, p. 309-313, 2020. 
SNOSWELL, C. L. et al. A cost-consequence analysis comparing patient travel, outreach, and telehealth clinic models for a specialist diabetes service to Indigenous people in Queensland. Journal of Telemedicine and Telecare, London, v. 25, n. 9, p. 537-544, 2019. DOI 10.1177/1357633X19873239. Disponível em: https://journals.sagepub.com/doi/abs/10.1177/1357633X19873239. Acesso em: 11 jun. 2020

SONG, X.; LIU, X.; WANG, C. The role of telemedicine during the COVID-19 epidemic in China-experience from Shandong province. Critical Care, London, v. 24, n. 1, p. 178, Apr. 2020. DOI: 10.1186/s13054-020-02884-9. Disponível em: https://ccforum.biomedcentral.com/articles/10.1186/ s13054-020-02884-9. Acesso em: 4 maio 2020.

MIRANDA, S. P. et al. Letter to the Editor "Incorporating Telehealth to Improve Neurosurgical Training During the COVID-19 Pandemic". World Neurosurgery, New York, v. 139, p. 728-731. July 2020. Disponível em: http://www.sciencedirect.com/science/article/pii/S1878875020310871. Acesso em: 22 jul. 2020.

SUN, S. et al. China empowers Internet hospital to fight against COVID-19. The Journal of Infection, v. 81, n. 1, p. e67-e68, 2020. DOI: https://doi.org/10.1016/j. jinf.2020.03.061. Disponível em: https://www.journalofinfection.com/article/ S0163-4453(20)30183-3/fulltext. Acesso em: 17 abr. 2020.

UNIVERSIDADE FEDERAL DA BAHIA - UFBA. EdgardigitaL. TeleCoronavírus atendeu cerca de 24 mil pessoas em 42 dias de operação. Salvador, 8 maio 2020. Disponível em: http://www.edgardigital.ufba.br/?p=16854. Acesso em: 10 maio 2020.

VIDAL-ALABALL, J. et al. Primary Care Professionals' Acceptance of Medical Record-Based, Store and Forward Provider-to-Provider Telemedicine in Catalonia: Results of a Web-Based Survey. International Journal of Environmental Research and Public Health. Basel, v. 17, n. 11, p. E4092, 2020. DOI: 10.3390/ ijerph17114092. Disponível em: https://www.mdpi.com/1660-4601/17/11/4092. Acesso em: 26 jun. 2020.

YE, J. Pediatric Mental and Behavioral Health in the Period of Quarantine and Social Distancing With COVID-19. JMIR Pediatrics and Parenting, Toronto, v. 3, v. 2, p. e19867, 2020. DOI: https://doi.org/10.2196/19867. Disponível em: https:// pediatrics.jmir.org/2020/2/e19867/. Acesso em: 11 jul. 2020. 
YAO, H. et al. Mitigating mental health consequences during the COVID-19 outbreak: Lessons from China. Psychiatry and Clinical Neurosciences, Carlton, v. 74, n. 7, p. 407-408, 2020. DOI: 10.1111/pen.13018. Disponível em: https://onlinelibrary.wiley.com/doi/full/10.1111/pcn.13018. Acesso em: 20 maio 2020.

WHITTEN, P.; ADAMS, I. Success and failure: a case study of two rural telemedicine projects. Journal of Telemedicine and Telecare. London, v. 9, n. 3, p. 125-129, 2003. DOI: 10.1258/135763303767149906. Disponível em: https:// europepmc.org/article/med/12877772. Acesso em: 11 jul. 2020.

WORLD HEALTH ORGANIZATION - WHO. Global diffusion of eHealth: Making universal health coverage achievable. Geneva: WHO, 2016. Disponível em: https://www.who.int/goe/publications/global_diffusion/en/. Acesso em: 24 abr. 2020.

WORLD HEALTH ORGANIZATION - WHO. Atlas: eHealth country profiles. Geneva: WHO, 2011. (Global Observatory for eHealth series, v. 1). Disponível em: http://whqlibdoc.who.int/publications/2011/9789241564168_eng.pdf. Acesso em: 5 jun. 2020.

WORLD HEALTH ORGANIZATION - WHO. The State Of Broadband: Broadband Commission for Sustainable Development. Geneva: ITU : United Nations Educational, Scientific and Cultural Organization, 2019a. Disponível em: https://www.itu.int/dms_pub/itu-s/opb/pol/S-POL-BROADBAND.20-2019-PDF-E. pdf. Acesso em: 4 jun. 2020.

WORLD HEALTH ORGANIZATION - WHO. WHO Guideline: recommendations on digital interventions for health system strengthening. Geneva: WHO, 2019b. Disponível em: https://apps.who.int/iris/bitstream/ handle/10665/311941/9789241550505-eng.pdf?ua=1. Acesso em: 4 jun. 2020.

WORLD HEALTH ORGANIZATION - WHO. Fifty-eighth World Health Assembly. Geneva, May 2005. Disponível em: https://apps.who.int/iris/bitstream/ handle/10665/20398/A58_2005_REC1-en.pdf?sequence=1\&isAllowed=y. Acesso em: 5 jun. 2020

WORLD HEALTH ORGANIZATION - WHO. COVID-19 Strategy Update. Geneva: WHO, 14 Apr. 2020. Disponível em: https://www.who.int/docs/default-source/ coronaviruse/covid-strategy-update-14april2020.pdf?sfvrsn=29da3ba0_19. Acesso em: 04 jun. 2020 
WORLD HEALTH ORGANIZATION - WHO. Use of appropriate digital technologies for public health: Report by the Director-General. 2017. Disponivel em: https://apps.who.int/iris/bitstream/handle/10665/274134/B142_20-en. pdf?sequence=1\&isAllowed=y. Acesso em: 13 jun. 2020.

WORLD HEALTH ORGANIZATION - WHO. Use of mobile wireless on public health. [s. I.], n. May, p. 1-4, 2016. Disponível em: https://apps.who.int/iris/ bitstream/handle/10665/274134/B142_20-en.pdf?sequence=1\&isAllowed=y. Acesso em: 15 jun. 2020.

ZHEN, H. et al. Telemedicine During the COVID-19 Pandemic: Experiences From Western China. Journal of Medical Internet Research, Toronto, v. 22, n. 5, p. e19577, 2020. Disponível em: https://www.jmir.org/2020/5/e19577/. Acesso em: 10 abr. 2020. 
